\title{
On the effect of fluid-structure interactions and choice of algorithm in multi-physics topology optimisation
}

\author{
David J. Munk ${ }^{\mathrm{a}, *}$, Timoleon Kipouros ${ }^{\mathrm{b}}$, Gareth A. Vio ${ }^{\mathrm{a}}$, Geoffrey T. \\ Parks $^{\mathrm{b}}$, Grant P. Steven ${ }^{\mathrm{a}}$ \\ ${ }^{a}$ The University of Sydney, Sydney, 2006, Australia \\ ${ }^{b}$ University of Cambridge, Cambridge, CB2 1PZ, United Kingdom
}

\begin{abstract}
This article presents an optimisation framework for the compliance minimisation of structures subjected to design-dependent pressure loads. A finite element solver coupled to a Lattice Boltzmann method is employed, such that the effect of the fluid-structure interactions on the optimised design can be considered. It is noted that the main computational expense of the algorithm is the Lattice Boltzmann method. Therefore, to improve the computational efficiency and to assess the effect of the fluid-structure interactions on the final optimised design, the degree of coupling is changed.

Several successful topology optimisation algorithms exist with thousands of associated publications in the literature. However, only a small portion of these are applied to real-world problems, with even fewer offering a comparison of methodologies. This is especially important for problems involving fluid-structure interactions, where discrete and continuous methods can provide different advantages.

The goal of this research is to couple two key disciplines, fluids and structures, into a topology optimisation framework, which shows fast convergence for multi-physics optimisation problems. This is achieved by offering a comparison of three popular, but competing, optimisation methodologies. The needs for the exploration of larger design spaces and to produce innovative designs make meta-heuristic algorithms less efficient for this task. A coupled analysis, where the fluid and structural mechanics are updated, provides superior results compared with an uncoupled analysis approach, however
\end{abstract}

\footnotetext{
*Corresponding author

Email address: david.munk@sydney.edu.au (David J. Munk)
} 
at some computational expense. The results in this article show that the method is sensitive to whether fluid-structure coupling is included, i.e. if the fluid mechanics are updated with design changes, but not to the degree of the coupling, i.e. how regularly the fluid mechanics are updated, up to a certain limit. Therefore, the computational efficiency of the algorithm can be considerably increased with small penalties in the quality of the objective by relaxing the coupling.

Keywords: topology optimisation, fluid-structure interactions, Lattice Boltzmann method, BESO, SIMP, level-set

\section{Introduction}

Topology optimisation of continuum structures has seen an exponential increase in publications (Munk et al. (2015)) since it was first proposed almost three decades ago (Bendsøe (1988)). Today it has matured to a level where it is becoming a common design tool used by industry. Here, the main idea is to find the optimal distribution of material in a predefined design domain considering an objective function and constraints. In the topology optimisation literature, one finds that a wide variety of objective functions have been considered, showing a diversity of application that spans to almost all fields of engineering and design (Sigmund (2001); Steven et al. (2000); Duhring et al. (2008)). However, design-dependent pressure loading problems are still uncommon (Picelli et al. (2015a); Munk et al. (2017a)), with little discussion on the effect of the degree of coupling on the design and no comparison between optimisation methods.

Traditional topology optimisation methods seek to find the maximum stiffness with a predefined fixed loading (Eschenauer and Olhoff (2001); Sigmund and Maute (2013); Deaton and Grandhi (2014)). However, there are many applications in which the load location and magnitude vary as the design changes during the optimisation process. Recently, the authors of this study developed a Bi-directional Evolutionary Structural Optimisation (BESO) algorithm that is coupled to a Lattice Boltzmann method (LBM) for the optimisation of design-dependent pressure loading problems (Munk et al. (2017a)). The method was applied to an industry design problem: namely, the design of micro fluidic mixers. It was found that the computational time required to solve such problems exceeded a reasonable level for use in the preliminary design stages (Munk et al. (2017a)). Furthermore, only a BESO 
algorithm was employed. Hence, this study proposes relaxing the degree of coupling between the LBM and finite element analysis (FEA) to quantify the impact of this coupling on the objective function value found and computational efficiency of the algorithm. Moreover, other types of topology optimisation methods, both continuous and discrete, are implemented into the framework. The results of the different optimisation methods indicate that they have different outcomes. Therefore, the advantages of the different optimisation methods are identified.

The examples considered in this study are further complicated by the Fluid-Structure Interactions (FSI) present between the structure being optimised and the flow, making the design dependent on the pressure loading from the fluid. The challenge in optimising a structure with an applied pressure load lies in determining the loading surface on which the pressure acts. This becomes more difficult for traditional density-based topology optimisation methods, such as Solid Isotropic Material with Penalisation (SIMP) (Rozvany et al. (1992)) and Homogenization (Bendsøe (1988)). In these methods, the structural boundaries, and hence loaded surfaces, are not explicitly defined due to the presence of intermediate density elements (Hammer and Olhoff (2000)). Therefore, in this study, a novel filter scheme is developed such that the structural boundary at each iteration is determined.

In this article, extended BESO, level-set and SIMP algorithms are applied to the design of micro fluidic mixers considering FSI. A three-dimensional (3D) LBM is used as the flow solver with two fluid species, extending beyond the basic two-dimensional (2D) Stokes flow used in the literature. Multiple optimisation techniques, both continuous and discrete, are compared, evaluating the benefits of these methods for design-dependent pressure-loaded problems. With the proposed framework, the design of structures subjected to fluid pressure loads can be easily implemented with high fidelity algorithms incorporated at the conceptual and detailed design phases, efficiently coupling multiple physical models. Furthermore, insight is given into the advantages and disadvantages of using different optimisation techniques.

The rest of this article is organised as follows. Sect. 2 outlines the necessary background and literature for the manuscript. Sect. 3 presents the governing equations for the fluid and structural models. Sect. 4 presents the topology optimisation problem and the various topology optimisation methods employed in this work. In Sect. 5, the methodology for coupling the multiple disciplines and extending the optimisation methods to the fluidstructure problem is outlined. The results from the three different topology 
optimisation algorithms are given in Sect. 6 for structural optimisation problems with design-dependent loads. Finally, Sect. 7 concludes the article.

\section{Background}

In the literature on continuous topology optimisation, significant effort to solve topology optimisation problems considering design-dependent pressure loads has been in the creation of the loading surface (Munk et al. (2017a)). One finds that several methods exist to achieve this; however, they can primarily be arranged into two groups. The first group seeks to identify a fluidstructure boundary and directly apply the loads onto the finite elements. Hammer and Olhoff (2000) suggest the use of Bezier spline functions for the identification of iso-density nodal points to obtain the boundary where the pressure will act. This method was improved upon by Du and Olhoff (2004a,b), where a modified technique for finding the density isolines is suggested. Fuchs and Shemesh (2004) also used Bezier curves, though they defined control points that are independent of density and are controlled by the optimiser. Recently, Lee and Martins (2012) improved upon the method of Du and Olhoff (2004b), by removing the need for the predefinition of isoline endpoints. Likewise, Gao and Zhang (2009) developed a pressure updating scheme for contact problems with solid weight pressure loading. Finally, Zhang et al. (2008) presented a boundary search scheme where the sensitivity of the loading to the element density can be ignored since the loads are determined from real element boundaries, rather than isolines. Alternatively, the second group of methods model the pressure loading with alternative physics or utilise mixed formulations to avoid explicitly defining a loading surface. Chen and Kikuchi (2001) used a fictitious thermal loading to simulate the pressure and employed a dryness coefficient to identify the fluid and solid regions. Similarly, Zheng et al. (2009) introduced a potential function modelled on the electric potential and applied a fictitious electric field. Alternative schemes have also been proposed to find design-dependent pressure loads using density-based methods. Bourdin and Chambolle (2003) used a fictitious liquid in a fluid-solid-void topology optimisation. They employed a perimeter penalisation technique to avoid homogenisation of the phases. Sigmund and Clausen (2007) modelled the fluid region as an incompressible hydrostatic fluid, introducing an extra design variable for each element. They determined the phase of the region using the two design variables. Similarly, Bruggi and Cinquini (2009) proposed a mixed equivalent formulation using 
another element approximation in order to avoid numerical difficulties due to the incompressible model assumption. Recently, Andreasen and Sigmund (2013) extended this method to topology optimisation of FSI problems in saturated poroelastic media. Thus, the literature shows that the classic element density-based topology optimisation algorithms become onerous when dealing with FSI coupled systems.

An alternative branch of topology optimisation, which lends itself to the application of design-dependent pressure loads, is based on discrete methods. One such method, BESO, has developed to the stage where it has been used by industry (Rozvany (2009)). The discrete update scheme present in evolutionary methods allows the use of separate modules for the fluid and structural domains with different governing equations. This overcomes a well-studied challenge associated with the classic density-based methods: dealing with moving multiphysics loads and interfaces. Therefore, discrete methods, such as BESO, offer great potential for applications in the areas of multiphysics optimisation. However, they are seldom found in the literature, likely due to their oscillatory convergence (Sigmund and Maute (2013); Munk et al. (2017b)). Possibly the first application of BESO to design-dependent problems can be found in (Yang et al. (2005)). Yang et al. (2005) applied evolutionary methods (Xie and Steven (1993)) to the design of structures, which included structural downward surface loads. They extend the BESO method to applications in fluid-loaded structural problems. Recently, Picelli et al. (2015a) extended this method to the application of general movable fluid-structure interfaces with design-dependent pressure loads. Later, Picelli et al. (2015b) applied this method to topology optimisation problems for frequency maximisation considering acoustic-structure interactions. Most recently, Munk et al. (2017a) coupled a BESO algorithm to a LBM for the design of micro fluidic mixers with the fluid-structural coupling present. They then extended this method to include multiple objective topology optimisation problems with design-dependent pressure loads (Munk et al. (2018)).

Level-set methods have the advantage that material boundaries are implicitly defined, thus they have also been applied to solve pressure loading problems (Sethian and Wiegmann (2000); Osher and Santosa (2001); Allaire et al. (2004); Liu et al. (2005)). These methods use boundary points as the design variables, deriving shape sensitivities to predict design changes. However, because the optimisation is based on the structural shape movements, they have been criticised for being dependent on the initial topology (Jenkins and Maute (2016)). Challis and Guest (2009) propose a level-set method 
for the optimisation of fluid flow. They show that the discrete nature of the optimisation problem leads to significant advantages over density-based topology optimisation algorithms. Furthermore, the no-slip boundary condition can be implemented directly, which is accurate and removes the need for interpolation schemes and continuation methods. This gives notable computational savings, since it only requires flow to be modelled in fluid regions. Topological changes can be incorporated into the level-set method by altering the level-set evolution equation to include topological sensitivity information (Burger et al. (2004); Amstutz and Andra (2006); He et al. (2007); Dunning and Kim (2013)). Zhou and Li (2008) apply such methods to the optimisation of steady-state Navier-Stokes fluid flows. They report on the computational expense of re-meshing in their level-set algorithm.

From this literature it is clear that, although methods for design-dependent pressure loads have been studied, most examples given are 2D, using simple Stokes flow solvers with linear pressure fields (Picelli et al. (2015a)). Research into density-based methods with design-dependent loading is limited to methods for determining the load surface (Lee and Martins (2012)). This is easily circumvented by using discrete algorithms; however, continuous approaches are more common in the topology optimisation literature, as they guarantee steady, monotonic convergence (Sigmund and Maute (2013)). This is further emphasised by the lack of literature on discrete algorithms being applied to design-dependent pressure loads for structural topology optimisation (Yang et al. (2005); Picelli et al. (2015a); Munk et al. (2017a)).

\section{Governing equations}

The problem considered in this work involves the static analysis of flexible structures in contact with two species of non-reacting isothermal and incompressible fluid. The structure is modelled using small strain and displacement theory of linearly elastic isotropic solids and the fluid domain is modelled through the use of a viscid and rotational LBM (Succi (2001)). This section briefly outlines the governing equations of the structural and fluid models.

\subsection{Structural model}

A fixed mesh linear static FEA is used as the structural model of this work, avoiding both fitted meshes and re-meshing, which is computationally 
inefficient and not always straightforward (Wang and Wang (2006)). Fournode shell elements, with membrane, bending and transverse shear stress are used to model the structure. The structural displacement, $\overrightarrow{\mathbf{u}}$, due to an applied load, $\overrightarrow{\mathbf{f}}$, can be found from:

$$
\overrightarrow{\mathbf{f}}=[\mathbf{K}] \overrightarrow{\mathbf{u}}
$$

where $\overrightarrow{\mathbf{u}}$ and $\overrightarrow{\mathbf{f}}$ are the nodal displacement and force vectors respectively. The stiffness distribution of the structure is defined by the global stiffness matrix $[\mathbf{K}]$. Therefore, Eq. 1 can be solved to get the structural displacements, $\overrightarrow{\mathbf{u}}$, for a given stiffness distribution, $[\mathbf{K}]$, as a result of an applied load, $\overrightarrow{\mathbf{f}}$. The FEA method, used in this study, was implemented by the authors and has been verified in previous studies (Munk et al. (2017a, 2018)).

The discussion of the finite element method is here kept brief. The authors advise the interested reader, who seeks further details of the finite element method, to consult the textbook of Chandrupatla and Belegundu (1997).

\subsection{Fluid models}

Since the first application of fluid dynamics in evolutionary (Steven et al. (2000)) and continuous (Borrvall and Petersson (2003)) topology optimisation, several different methods for modelling the fluid domain have been proposed. Typically, a fluid problem is solved by either finite volume, finite element or discontinuous Galerkin methods (Laniewski-Wollk and Rokicki (2016)). However, these methods have a number of difficulties when solving for complex 3D geometries and multiphase flows with variable complex interfaces, since they require fine-body-fitted meshes. Therefore, structural topology optimisation with fluid pressure loads are often limited to simple Stokes flow models and 2D cases (Picelli et al. (2015a)).

Conversely, Lattice Boltzmann methods solve a discrete Boltzmann equation on a Cartesian grid. Therefore, the LBM can easily handle complex geometries (Pan et al. (2006)). The LBM has been previously applied to the optimisation of channel flows (Challis and Guest (2009); Kreissl et al. (2010); Makhija et al. (2012)), and recently to the optimisation of structures subjected to design-dependent loads (Munk et al. (2017a, 2018)). Therefore, in this work, a 3D LBM is used as the fluid model. The LBM has been validated against Navier-Stokes simulations (Djenidi and Moghtaderi (2006)) and experimental analysis (Moghtaderi et al. (2006)), hence proving the accuracy of this method. 
The fundamental concept behind the LBM is the construction of kinetic models, based on Newton's laws, that incorporate the essential physics of the microscopic processes, so that these processes are modelled correctly. Therefore, the fluid is considered as a finite number of molecules, whose motion is governed by Newton's laws of dynamics. The LBM solves a discretised Boltzmann equation, known as the Lattice Boltzmann Equation (LBE). This work uses the D3Q19 lattice, meaning three dimensions and 18 moving particles per node with one rest node. The LBE represents the macroscopic properties by incorporating the velocity distribution function, $f(x, t, \gamma)$, and is expressed as follows:

$$
S h \frac{\partial f}{\partial t}+\gamma \cdot \nabla f=Q(f)
$$

where $S h$ is the Strouhal number, $t$ represents time, $x$ and $\gamma$ are the gas particle position and velocity respectively, and $Q$, which is known as the collision operator, models the effect of collisions between the particles. For the simulations in this work, the Bhatnagar-Gross-Krook (BGK) collision model (Bhatnagar et al. (1954)) is employed.

The fundamental concept of the LBM is the discretisation of the infinite set of particle velocities, $\gamma$, in Eq. 2. From this the calculation of macroscopic quantities, by solving the moments of a finite number of velocity distribution functions, can be obtained as solutions to the Navier-Stokes equations. Thus, the discretisation of the LBE (Eq. 2) using the BGK collision model in time and space at the lattice site $x$ is found from:

$f_{\alpha}\left(x+\gamma_{\alpha} \cdot \Delta t, t+\Delta t\right)-f_{\alpha}(x, t)=-\frac{1}{\tau_{B}} \cdot\left[f_{\alpha}(x, t)-f_{\alpha}^{e q}(x, t)\right] \quad$ for $\alpha=0, \ldots, 18$

where $\tau_{B}$ is the dimensionless relaxation time, $\Delta t$ is the time step and $\alpha$ is the number of particles per node. The density, $\rho$, and fluid velocity, $v$, are obtained from the moments of the velocity distribution functions, as follows:

$$
\rho=\sum_{\alpha=0}^{18} f_{\alpha} \quad \text { and } \quad v=\frac{1}{\rho} \sum_{\alpha=0}^{18} \gamma_{\alpha} f_{\alpha}
$$

Therefore, Eq. 3 is solved to simulate the fluid dynamics of the system, the resulting velocity distribution functions are then substituted into Eq. 4 to get the macroscopic properties. The total number of iterations used for the LBM simulations is 4000, since stability has been demonstrated and validated in (Munk et al. (2017a); Tsotskas et al. (2015)). 
The authors advise readers who are interested in the LBM and seek further discussion to find the textbook by Succi (2001).

\section{Topology optimisation}

This work is concerned with the compliance minimisation of structures under fluid pressure loading with volume constraints. The objective is to find the distribution of a given amount of material to obtain a structure with maximum stiffness. Therefore, the topology optimisation problem for this case can be stated as:

$$
\begin{array}{cl}
\text { Minimise: } & \frac{1}{2} \overrightarrow{\mathbf{u}}^{T}[\mathbf{K}] \overrightarrow{\mathbf{u}} \\
\text { subject to: } & {[\mathbf{K}] \overrightarrow{\mathbf{u}}=\overrightarrow{\mathbf{f}}} \\
& \sum_{i=1}^{n} x_{i} \leq V \\
& \mathbf{x}=[0,1]
\end{array}
$$

where $\mathbf{x}$ is the vector of design variables, $x_{i}, n$ is the total number of elements in the model and $V$ is a predefined volume fraction. For discrete algorithms (Sections 4.1 and 4.2 ) the design variable, $x_{i}$, is forced to become discrete, taking on the endpoints of the range, where $x_{i}=1$ represents solid and $x_{i}=0$

represents void or fluid material. In the continuous algorithm formulation (Sect. 4.3), the design variables are continuous and hence $0 \leq x_{i} \leq 1$.

\subsection{Evolutionary Structural Optimisation}

The Evolutionary Structural Optimisation (ESO) method was introduced as a monotonic algorithm, meaning elements could only be removed from the design domain (Xie and Steven (1993)). These early heuristic methods are based on the structure gradually evolving towards the optimal design by the successive elimination of inefficient material, defined as material that exhibits low levels of stress when a load is applied, from the initial, oversized, structure (Xie and Steven (1997)). Although the ESO method has been applied to a wide range of problems (Xie and Steven (1996); Steven et al. (2000)), it is limited by only removing material from the structure. This results in two shortcomings. Firstly, the initial model must be significantly over-designed, and secondly, if material is prematurely removed, it 
cannot be recovered (Munk et al. (2015)). From these limitations, subsequent ESO methods, now known as BESO methods, were developed, which allow material, if deemed beneficial, to be re-admitted to the design domain (Querin et al. (1998)). Modern BESO algorithms are convergent and meshindependent (Huang and Xie (2007)), simultaneously removing and adding material from and to the design domain until the constraints and a convergence criterion are satisfied. A further improvement to BESO methods introduced the use of soft material to model the void elements in the FEA (Huang and Xie (2009)). This method became known as soft-kill BESO, with the former method being hard-kill BESO. In this article, the proposed methodology uses a soft-kill BESO method. This contrasts to the work in (Picelli et al. (2015a, 2017)), which used a hard-kill BESO method, where the fluid and structural models were solved together, and soft elements could therefore not be handled. The work outlined in this article takes advantage of the different governing equations (Sect. 3), using separate modules for the fluid and structural domains, with information being passed between the two modules (Sect. 5) to implement a coupled solution. For a detailed overview of the BESO algorithm more information can be found in the latest textbook (Huang and Xie (2010)) and review papers Munk et al. (2015); Xia et al. (2016) on the subject.

\subsection{Level-set methods}

The Level-Set Method (LSM) was developed by Osher and Sethian (1988) to model moving boundaries, typically for the evolution of interfaces in multiphase flows. The LSM concept was later extended to topology optimisation by Haber and Bendsøe (1998) for the description of the geometry during the optimisation process. Originally, the LSM solved a Hamilton-Jacobi type equation to update the level-set function (Allaire et al. (2004)), thus limiting LSMs to shape optimisation, since only the present structural boundaries are updated. A mechanism to create new holes inside the structure was first presented by Eschenauer et al. (1994). Termed the bubble method, they propose shape optimisation augmented with a hole-positioning criterion to insert a new hole in the material domain. Allaire et al. (2005) introduced the bubble method to the level-set-based topology optimisation algorithm in order to allow topological changes. However, the introduction of new holes during the optimisation process is facilitated by predefined parameter values, which have been proven to be difficult to define, as they often show a strong dependency on the optimal configurations obtained (Yaji et al. (2014)). Dun- 
ning and Kim (2013) presented a new method for hole insertion in LSMs, which utilises a secondary level-set function to facilitate new hole generation. They demonstrate that, because the update of the secondary function is connected to the primary level-set function, a meaningful link between boundary optimisation and hole creation is present. Recently, Yamada et al. (2010) proposed a new LSM, modelled off the concept of the phase field method, and using a reaction-diffusion equation to update the level-set function. This method permits topological changes, and has been shown to be mesh-independent and to have minimal dependency on initial configurations (Yamada et al. (2010)). However, such a method has not been extended to topology optimisation with a design-dependent pressure load. This article uses the LSM proposed by Yamada et al. (2010) as the level-set-based topology optimisation algorithm. The interface between the solid and void/fluid phase is implicitly defined by the iso-contours of the level-set function. The fluid and structural domains are therefore solved separately, similar to the evolutionary optimisation algorithm (Sect. 4.1). Recently, this method was coupled with an LBM for the optimisation of fluid dynamics problems (Yaji et al. (2014)), demonstrating the suitability of such methods for use with the LBM. The application of the LBM to the optimisation of fluid flows with a meta-heuristic algorithm has also been demonstrated (D'Ammaro et al. (2011)). This work builds on this research by applying the same methods, but to coupled structural and fluid flow mechanics. Further details on the LSM for topology optimisation can be found in the latest review article (van Dijk et al. (2013)) on the subject.

\subsection{Solid Isotropic Material with Penalisation}

The SIMP method is a material power law approach that assigns a design variable to each element to model the fictitious microstructure, the properties of which are given by a continuous material model. SIMP was originally introduced (Bendsøe (1989); Zhou and Rozvany (1991); Mlejnek (1992)) as an easy but artificial way of reducing the complexity of the earlier Homogenization approach (Bendsøe (1988)) and to improve its convergence to 1-0 solutions. However, since then, a physical justification of SIMP has been provided (Bendsøe and Sigmund (1999)), and it has gone on to become one of the most popular techniques for structural topology optimisation (Rozvany (2001)). A similar method to SIMP, known as Rational Approximation of Material Properties (RAMP) (Stolpe and Svanberg (2001)), has been suggested to alleviate the non-concavity of the original SIMP material in- 
terpolation scheme and hence ensure convergence to 1-0 solutions. However, this scheme has little influence in practical problems (Sigmund and Maute (2013)), rather it has been used to alleviate fictitious low density modes in dynamic problems (Pedersen (2000)). Nevertheless, both methods require the proper choice of the penalisation factor, $p$, for the problem being solved. The realisation that the compliance minimisation problem becomes convex for $p=1$ motivated the use of continuation approaches that start with $p=1$ and gradually increase the penalisation during the optimisation process (Rozvany (2009)). This will often result in convergence to better designs (Sigmund and Maute (2013)). However, in general, the continuation method cannot guarantee convergence to 1-0 structures. The modern SIMP method is meshindependent and convergent (Sigmund and Petersson (1998)), though final structures often contain some intermediate material. In this work, the SIMP method without continuation is employed. A novel filter, which conserves the volume fraction of the structure (Sect. 5), is implemented to couple the SIMP topology optimisation algorithm with the LBM flow solver. More details on SIMP methods can be found in the latest textbook (Rozvany and Lewinski (2014)) and review paper (Deaton and Grandhi (2014)) on the topic.

\section{Fluid-structure interactions}

This section describes the extensions made to the topology optimisation algorithms (Sect. 4) such that design-dependent pressure loads are considered in the optimisation routine. First, the coupling framework, which shows how the fluid and structural solvers are coupled, is outlined and methods for loosening the coupling to increase computational efficiency described (Sect. 5.1). The derivation of the updated sensitivity number for a compliance minimisation problem with a design-dependent fluid pressure load is then given (Sect. 5.2). Finally, the filter scheme, which can be used for coupling continuous topology optimisation algorithms with a LBM fluid solver, is given (Sect. 5.3). It is noted that this filter scheme serves the purpose of determining the loaded surface for the fluid pressure. The literature shows several different techniques (Sect. 2) for this purpose; however, to the best of the authors' knowledge such a method, as proposed herein, has not been suggested before. 


\subsection{Coupling framework}

The numerical framework, which couples the topology optimisation algorithms (Sect. 4) with the Lattice Boltzmann flow solver (Sect. 3.2) and finite element analysis (Sect. 3.1), is shown in Fig. 1. The problem is defined, setting the initial geometry and boundary conditions for the test case. The geometry and boundary conditions are then input into the flow solver, which outputs the pressure, and ultimately the forces, being applied to the structure. The current topology and loads are passed onto the Finite Element Method (FEM) module, which defines the structural boundary conditions and outputs the displacements and compliance of the structure. This is passed onto the topology optimisation algorithm, which calculates the sensitivities and updates the topology of the structure for the next iteration.

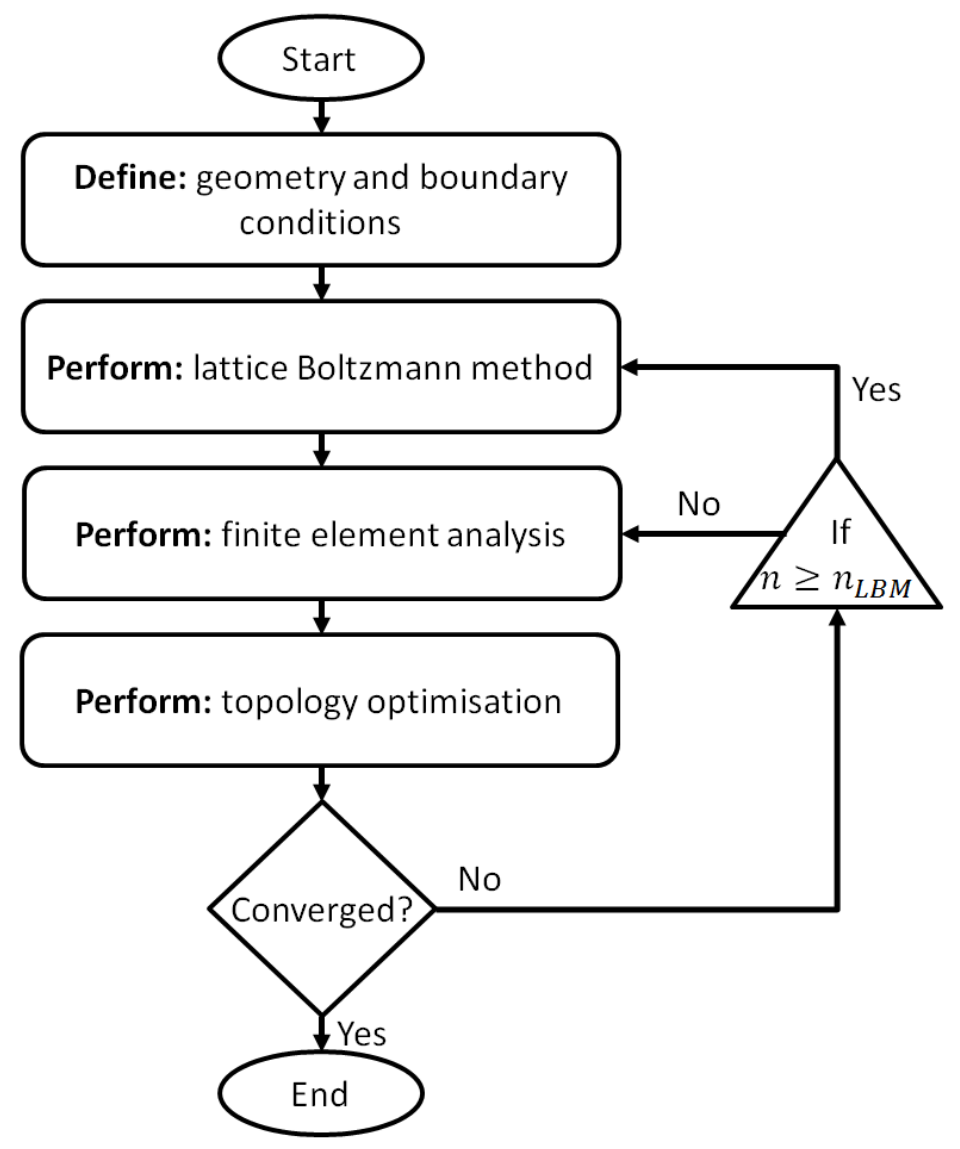

Figure 1: Numerical framework for coupled LBM-topology optimisation. 
There are two loops present in the numerical framework (Fig. 1). The first is between the topology optimisation algorithm and the FEM module, which is performed at every iteration in the optimisation process. The second loop passes the updated topology back through the LBM flow solver. Due to the computational cost of the LBM solver, the second loop can be restricted to only be performed every $n_{L B M}$ iterations. This is a predefined parameter, which determines the amount of change in the topology required before the fluid dynamics and pressure loads are updated. To determine the effect of $n_{L B M}$ on the objective and computational efficiency of the algorithm, a tradeoff between reduction in compliance and computation time is given in the results section (Sect. 6). Further, to reduce the computational burden of the coupled LBM-topology optimisation method, identical grids are used in the LBM and FEA analyses, thereby avoiding complex, time-inefficient, spline methods to transfer the loads between the structural and fluid modules and re-meshing of the domains (Zhou and Li (2008)).

\subsection{Sensitivity analysis: design-dependent pressure load}

In design-dependent load problems, by definition, modifying the structure leads to changes in the load vector. Therefore, the change in the load vector must be considered in the sensitivity analysis. Thus, from the definition of the optimisation problem (Eq. 5) the sensitivity analysis for compliance minimisation can be modified, such that the change in the load vector is considered, as follows (Yang et al. (2005); Picelli et al. (2015a); Munk et al. $(2017 \mathrm{a}))$ :

$$
\alpha_{e}=\frac{1}{2} p x_{i}^{p-1} \overrightarrow{\mathbf{u}}_{e}^{T}[\mathbf{K}]_{e} \overrightarrow{\mathbf{u}}_{e}+p x_{i}^{p-1} \overrightarrow{\mathbf{u}}_{e}^{T} \Delta \overrightarrow{\mathbf{f}}_{e}
$$

where $\Delta \overrightarrow{\mathbf{f}}_{e}$ is the change in the element load vector between optimisation loops. Taking the isoparametric bilinear elements used in this work, the change in the load vector of one element for a fluid pressure load is found by:

$$
\Delta \overrightarrow{\mathbf{f}}_{e}=\frac{1}{4} P_{i} A_{i}\{1,0,0,0,0,0,1,0,0,0,0,0, \ldots, 0\}_{24 \times 1}^{T}
$$

where $P_{i}$ is the pressure load on the element and $A_{i}$ is the elemental area. Eq. 7 makes the assumption that the flow travels in the positive $x$ direction, and that the structure is perpendicular to the flow. If the flow is travelling in another direction, or if the structure is not aligned perpendicular to the flow, then the load direction vector in Eq. 7 must be updated to match the 
loading conditions. However, it is noted here that when solving problems with design-dependent pressure loads in three dimensions, the load will always act perpendicular to the structure. Hence, the magnitude of the load and the surface it acts on change, but the direction of the load remains constant. Consequently, a coupling matrix is not required (Picelli et al. (2015a)), since the pressure load is always perpendicular to the surface (Fuchs and Moses $(2000))$.

\subsection{SIMP filter scheme}

To couple the fluid and structural domains in the topology optimisation process the updated geometry is input into the LBM flow solver to determine the updated fluid dynamics (Sect. 5.1). However, for continuous algorithms, such as SIMP (Sect. 4.3), the geometry is not explicitly defined. Therefore, an extra complication is present in such algorithms, since the loaded surface is unknown (Sect. 1). Hence, a method is developed here, like those in the first group of the literature (Sect. 2), which identifies the fluid-structure boundary and directly applies the loads to the structure (Hammer and Olhoff (2000); Du and Olhoff (2004b); Lee and Martins (2012)).

The method is similar to that initially proposed by Hammer and Olhoff (2000), with further improvements suggested by Du and Olhoff (2004a,b) and Lee and Martins (2012). Hammer and Olhoff (2000) introduced a parameterised iso-volumetric density curve to determine the variable loading surface. The pressure load can then be applied to this curve, where the nodal forces are calculated in the elements that intersect the curve. Du and Olhoff $(2004 \mathrm{a}, \mathrm{b})$ introduce constraints which avoid disconnected structures or structural islands being formed by the iso-volumetric density curve. They suggest not only using the density variables of the current iteration, but also the density variables from the previous iteration to improve the stability in the identification of the loading surface (Du and Olhoff (2004a)). Lee and Martins (2012) showed that this method relies heavily on the isoline from the previous iteration, since it assumes that the material distribution does not vary greatly between iterations. Therefore, they suggest the use of a predefined void area, the position of which is used to determine the isoline for each iteration (Lee and Martins (2012)).

The method introduced herein is based on those proposed by Du and Olhoff (2004a) and Lee and Martins (2012), adapted for 3D structures. The volume fraction between iterations is conserved (Fig. 2). Therefore, the isoline is determined by finding the lowest density such that the ratio of the 
number of elements with a density equal to or greater than the isoline to the number of elements in the design domain is equal to the volume fraction (Fig. 2a). Hence, the structural boundary becomes the outline produced by this isoline (Fig. 2b). The design variables are ranked in ascending order, $\overline{\mathbf{x}}=\operatorname{ascend}(\mathbf{x})$, and the density for the isoline is then found by:

$$
x_{\text {isoline }}=\overline{\mathbf{x}}_{\left(V_{D}-V\right)}
$$

where $V_{D}$ is the volume of the design domain. The elements that have a density below the isoline, $x<x_{\text {isoline }}$, become the void area for that iteration (Lee and Martins (2012)). In this way, the structure can be explicitly defined and passed through the LBM flow solver, to update the fluid dynamics and pressure loads (Fig. 2c). The new pressure loads are then applied to the original topology, i.e. before the isoline filter scheme, in the next iteration of the optimisation process (Fig. 2a). This process is shown graphically for one optimisation iteration in Fig. 2.

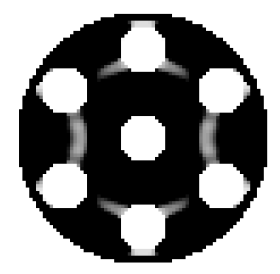

(a) Optimisation

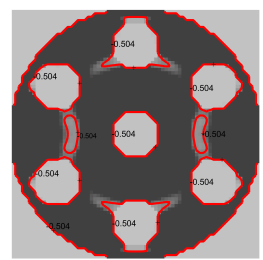

(b) Isoline

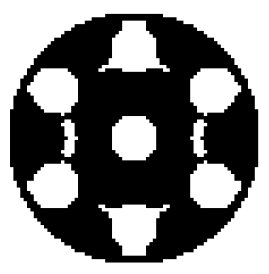

(c) LBM

Figure 2: Determining the loaded surface for SIMP topology optimisation.

\section{Results and discussion}

This section presents the results of the three different topology optimisation algorithms (Sect. 4) for the structural optimisation problem with designdependent loads. First, the case study analysed in this work is detailed. This is followed by the results of the algorithms without fluid-structure coupling, which present a baseline test case for the coupled results. Finally, the coupled results are given along with a trade-off of the improvement in the objective versus the computational expense of the algorithm. 


\subsection{Case study}

The model used in this study is a baffled micro-reactor, as depicted in Fig. 3. The model consists of a tubular vessel fitted with a fuel inlet tube, located co-axially in the main vessel, and a multi-holed baffle plate through which the oxidiser is introduced. The fluid domain and layout of the microreactor model are shown in Fig. 3.

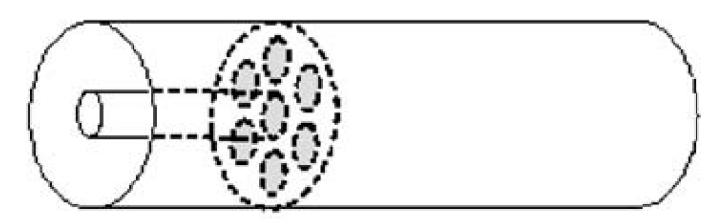

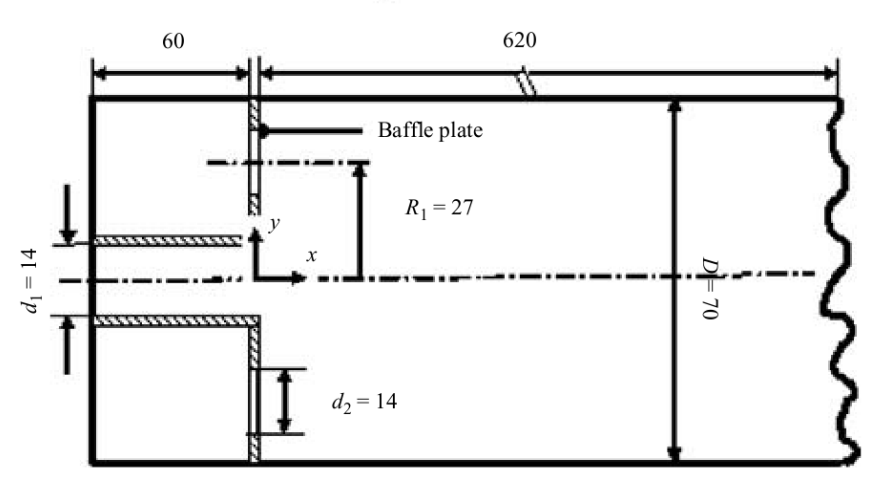

(a) Fluid domain and layout of micro-reactor model

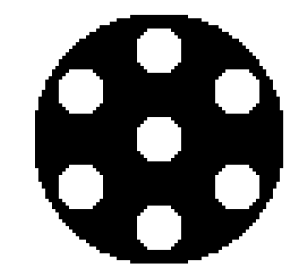

(b) Multi-holed baffle plate

Figure 3: Baffled micro-reactor case study (Tsotskas et al. (2015))

The dimensions of the fluid domain (Fig. 3) are expressed in LBM nodes, where the dimensions of the lattice are $680 \times 73 \times 73$ lattice units, with additional nodes used for the wall, in the $x, y$ and $z$ directions, respectively. The baffle is located 60 lattice units downstream of the flow inlet (Fig. 3). The imposed inlet conditions are the velocities of the flow in the inlet tube and annulus area. At the outlet a convective boundary condition is applied, based on the velocity. The no-slip condition at the walls is implemented by modelling them as full-way bounce-back. The mass flow rate between the inner tube and annulus is set to $5 \%$ to mimic the experiments performed by Moghtaderi et al. (2006).

In this section, the topology optimisation algorithms (Sect. 4) are applied to the multi-holed baffle plate (Fig. 3b) to maximise its stiffness for a given 
volume fraction. FEA (Sect. 3.1) is therefore performed on the baffle only. The plate is modelled using four-node quadrilateral elements, with all six degrees of freedom active, so membrane, bending and transverse shear stresses are present. A clamped boundary condition, i.e. all six degrees of freedom are restrained, is applied along the boundary of the baffle. The boundary of the central hole is designated as non-designable for the topology optimisation, since this is determined by the fuel line and inlet conditions, which have been constrained in the fluid domain (Fig. 3a) to be identical to the previous numerical (Djenidi and Moghtaderi (2006)) and experimental (Moghtaderi et al. (2006)) studies.

\subsection{No fluid-structure coupling}

First, the problem with no feedback to the fluid solver is analysed, thus reducing it to a standard topology optimisation problem with a fixed loading (Sigmund and Maute (2013); Deaton and Grandhi (2014)). The LBM is only applied once on the initial structure to obtain the pressure loads, which remain unchanged throughout the optimisation process. This represents the simplest (and hence most computationally efficient) case, but probably the least accurate. The results of this section are used as the benchmark for the design-dependent pressure loads (Sect. 6.3). For the sake of brevity, only the results for the LSM (Sect. 4.2) will be shown in detail here, since the effect of the regularisation parameter, $\varphi$, is studied. However, the final results for the BESO and SIMP algorithms will be given for comparison with the LSM and the design-dependent loading results. Since a standard topology optimisation problem is being solved, the results for the SIMP and BESO algorithms are comparable to the LSM. A comparison for fixed and designdependent pressure loads in two dimensions was given by $\mathrm{Du}$ and Olhoff (2004a).

The initial structure is shown in Figs. 3c and $4 \mathrm{a}$. The regularisation parameter, $\varphi$, and volume constraint iteration number, $n_{v o l}=50$, are defined before the level-set topology optimisation algorithm (Sect. 4.2) is applied. To examine the effect of the regularisation parameter, three different degrees of diffusivity are imposed by setting $\varphi=10^{-4}, 10^{-5}$ and $10^{-6}$.

For all three cases (Fig. 4), the final topology shows a more complex structure, creating load paths to increase the stiffness of the baffle. The results show that more complicated topologies, i.e. smaller holes, are obtained when $\varphi$ is set to smaller values. All six holes from the original structure have been maintained; however, the shape of the holes has become more 


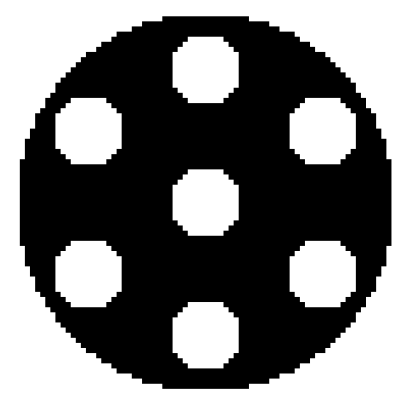

(a) Initial

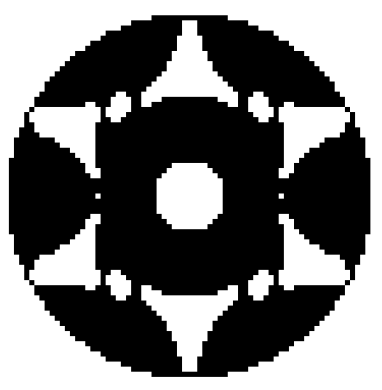

(c) $\varphi=10^{-5}$

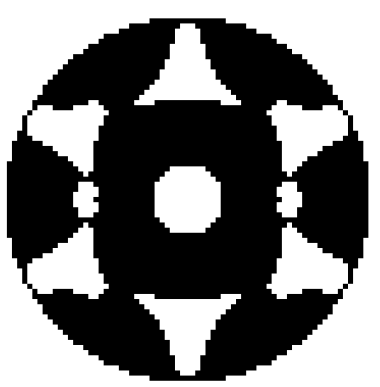

(b) $\varphi=10^{-4}$

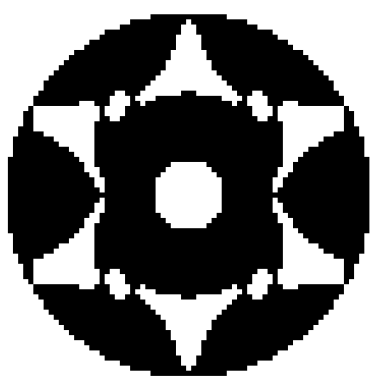

(d) $\varphi=10^{-6}$

Figure 4: Initial and final topologies for the baffle with fixed loading for LSM.

triangular. Nevertheless, for all cases, smaller additional holes have been generated. The strain energy distributions in the initial and final topologies are given in Fig. 5 .

The initial strain energy distribution shows several concentrated regions around the holes, where the overall strain energy is significantly higher compared to the rest of the structure (Fig. 5a). Most of the material in the baffle is therefore not being used efficiently. In comparison, the optimised topologies significantly reduce these concentrated zones (Fig. 5), especially if the centre hole is ignored, since this region is non-designable (Sect. 6.1). The material is being used more effectively in the optimised structures, as there are fewer zones of energy concentration. The optimiser is clearly able to improve the structural design of the baffle, even for a constant loading, by reducing the compliance and spreading out the loads more evenly through 


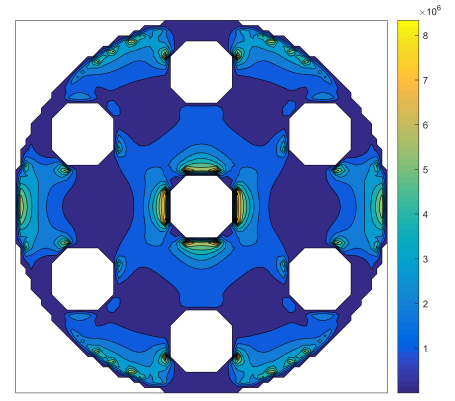

(a) Initial

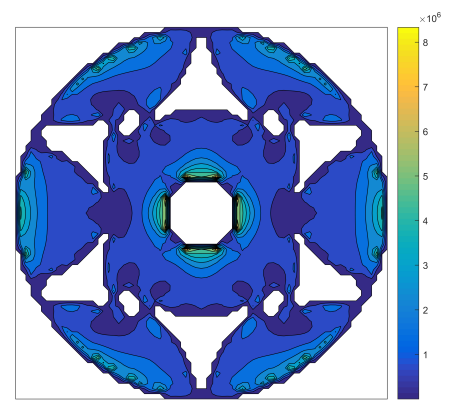

(c) $\varphi=10^{-5}$

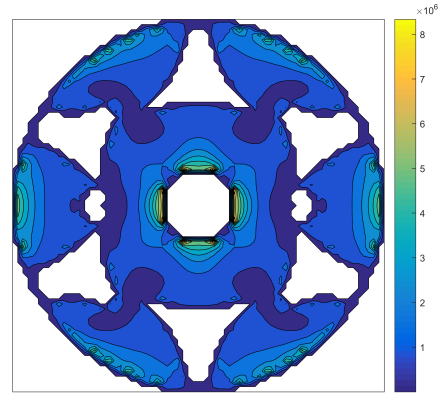

(b) $\varphi=10^{-4}$

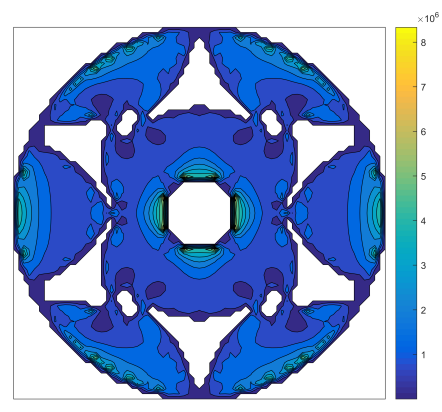

(d) $\varphi=10^{-6}$

Figure 5: Strain energy distributions for the baffle with fixed loading for LSM.

improved load paths.

The convergence histories for the different cases are given in Fig. 6. Due to the addition of the regularisation term, $\varphi$, it is not possible to guarantee that the objective function will decrease monotonically (Yamada et al. (2010)). Since the volume constraint is relaxed for the first 50 iterations, structure is initially added such that the volume is greater than the constraint, lowering the compliance below the minimum achieved. Once the optimiser has performed 50 iterations, the volume constraint is met, and the structure converges to a final design (Fig. 6). The jumps observed in the objective (Fig. 6) are a result of multiple holes combining to form one large hole. This behaviour is characteristic of discrete methods (Sigmund and Maute (2013)). Also, it is noted that a more erratic convergence history occurs for higher values of $\varphi$. Higher degrees of diffusivity, i.e. large $\varphi$, result 


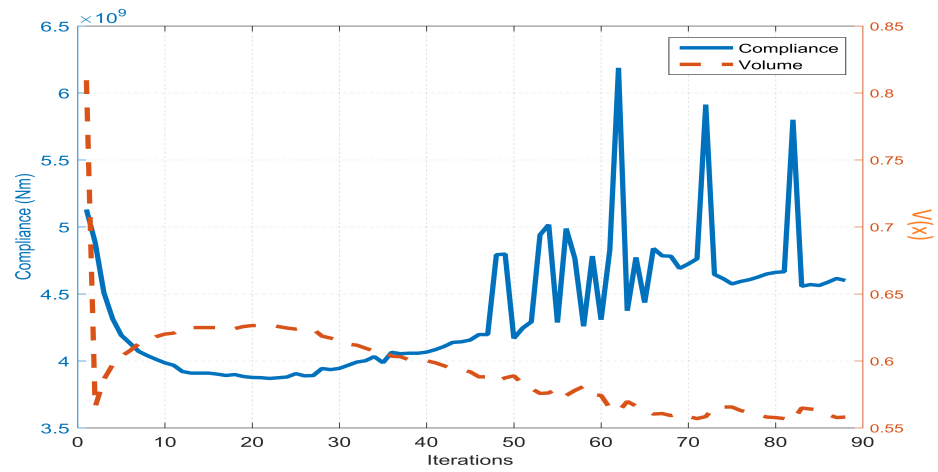

(a) $\varphi=10^{-4}$

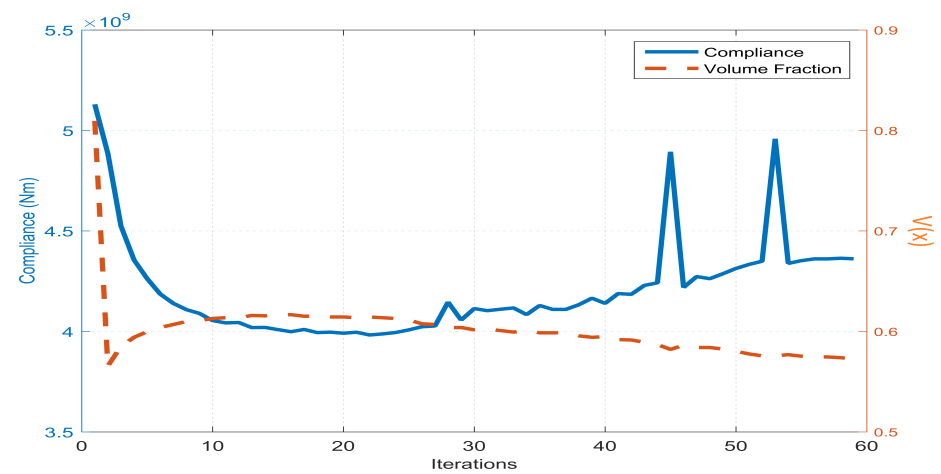

(b) $\varphi=10^{-5}$

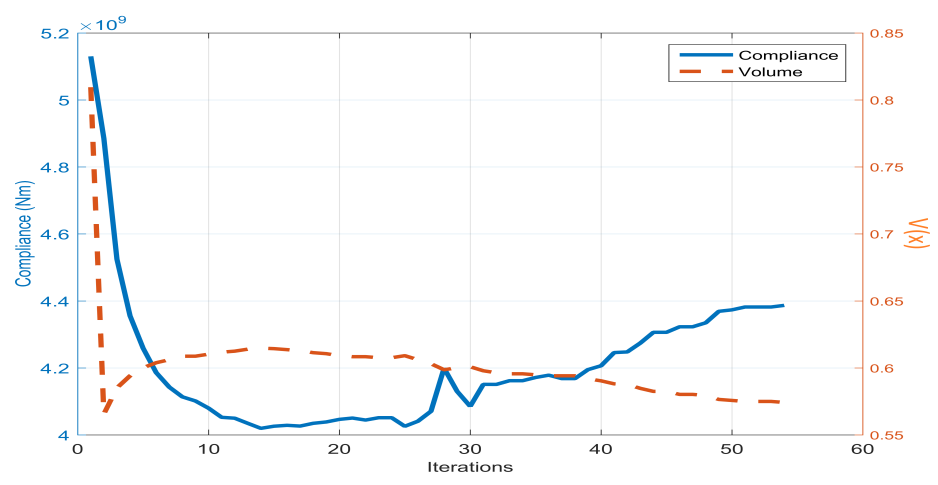

(c) $\varphi=10^{-6}$

Figure 6: Convergence histories for the baffle with fixed loading using the LSM algorithm. 
in the formation of larger holes, since less complicated (small hole) geometries are not permitted. This leads to more holes combining, which causes the large jumps in the objective. Thus, the regularisation parameter, $\varphi$, must be low enough to avoid severe oscillations. The results for the different cases are summarised in Table 1.

Table 1: Summary of results for the multiple degrees of diffusivity.

\begin{tabular}{rrr}
\hline Diffusivity $(\varphi)$ & Minimum compliance $(\mathrm{Nm})$ & Iterations \\
\hline $10^{-4}$ & $4.60 \times 10^{9}$ & 88 \\
$10^{-5}$ & $4.36 \times 10^{9}$ & 59 \\
$10^{-6}$ & $4.39 \times 10^{9}$ & 54
\end{tabular}

The initial structure has a compliance of $5.13 \times 10^{9} \mathrm{Nm}$, so the compliance has been reduced by approximately $10 \%, 15 \%$ and $14 \%$ for $\varphi=10^{-4}$, $10^{-5}$ and $10^{-6}$, respectively. Further, the larger diffusivity results in a slower convergence, due to the constraint on the allowable hole size resulting in bigger changes in topology between iterations. However, once the regularisation parameter is below $\varphi=10^{-5}$, the convergence rate starts to become constant. From this analysis, a regularisation parameter of $\varphi=10^{-5}$ seems ideal, since it produces a fast convergence and a final topology with the lowest compliance. Therefore, the LSM used in Section 6.3 also uses $\varphi=10^{-5}$.

As mentioned at the start of this section, a short comparison with the BESO and SIMP methods will be given for the constant loading case. The soft-kill BESO algorithm is able to reduce the compliance by $14 \%$ to $4.39 \times 10^{9}$ Nm. The optimisation history of the BESO algorithm is given in Fig. 7.

The sequence takes 60 iterations to converge to a final solution. The optimisation history is not monotonic (Fig. 7). This is expected as the BESO algorithm is discrete, and therefore the topology update is discrete. However, the results are comparable with the LSM for $\varphi \leq 10^{-5}$ (Figs. 6b and 6c). This is not surprising since, in its simplest form, the BESO algorithm is essentially the same as the LSM with a very small diffusivity, as any topology is allowed. This is seen by comparing the convergence histories of the LSM and BESO algorithms (Figs. 6 and 7), since, as the diffusivity is reduced, the convergence becomes more stable and smooth, similar to the convergence of the BESO algorithm. Therefore, for a simple topology optimisation problem with a constant loading, the LSM and BESO algorithms are comparable, producing similar final topologies. 


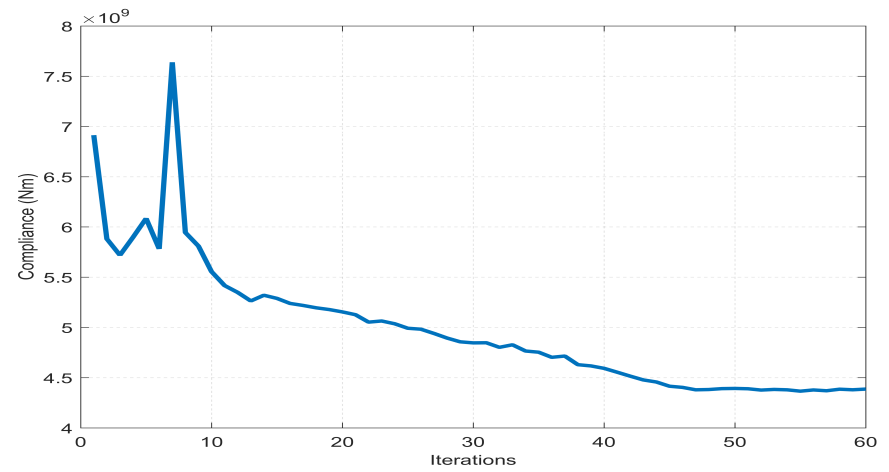

Figure 7: Convergence history for the baffle with fixed loading using the BESO algorithm.

Finally, the continuous SIMP algorithm is applied to the constant loading problem. A reduction in the compliance of $16 \%$, from $5.13 \times 10^{9} \mathrm{Nm}$ to $4.33 \times 10^{9} \mathrm{Nm}$, is achieved (Fig. 8).

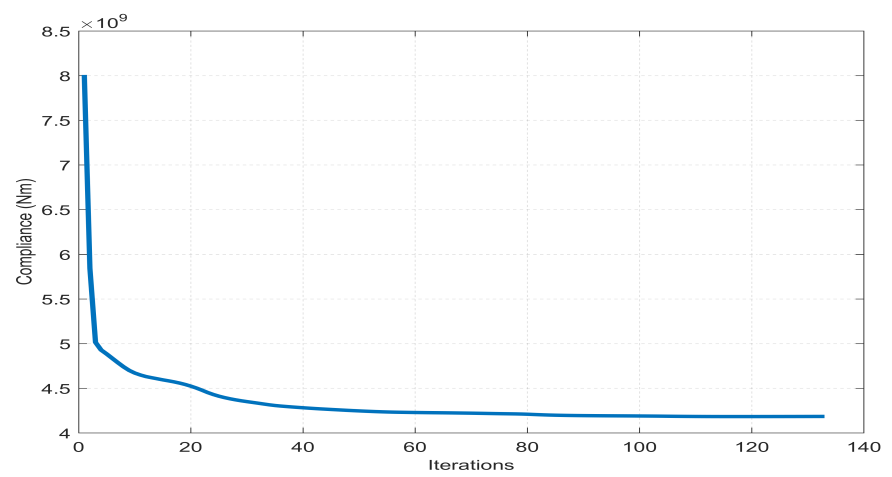

Figure 8: Convergence history for the baffle with fixed loading using the SIMP algorithm.

The sequence takes 133 iterations to converge; however, the last 50 iterations reduce the objective by only $1 \%$, from $4.38 \times 10^{9} \mathrm{Nm}$ to $4.33 \times 10^{9} \mathrm{Nm}$. This is because of the penalty exponent in the SIMP algorithm (Sect. 4.3), which penalises the intermediate material, driving the solution towards a solid-void topology. Thus, these final iterations are removing the intermediate material. Further, the optimisation history is monotonic, without any jumps in the objective. Therefore, the SIMP algorithm is able to guarantee a stable convergence, with the lowest final objective. Nevertheless, the final structure contains some intermediate material, which is not possible in the 
LSM and BESO algorithms.

Hence, for the constant loading problem, all the results are comparable, except possibly the LSM with $\varphi=10^{-4}$, which suggests the regularisation parameter is too high in this case. The final designs given by the discrete algorithms have a final compliance within $1 \%$ of each other, with the continuous algorithm achieving a slightly better final design, since intermediate structure is permitted. The improvement gained by the optimisation is significant as the baffle has become $15 \%$ (or $16 \%$ with intermediate material) more stiff compared with the original design. However, this was done without updating the pressure loads on the structure, meaning it is optimised for the initial load case and not the actual load case, which develops with the topology. Further, the fluid-structure interactions are not being considered as the flow physics are not updated with the structure. Thus, to determine the real physical compliance of the uncoupled designs, the loads must be recalculated for the final topologies. The final topologies are therefore run back through the LBM, and the physical compliance is calculated. This shows that the final compliance with the updated loads is reduced by $9 \%$ to $4.71 \times 10^{9}$ $\mathrm{Nm}$ from $5.13 \times 10^{9} \mathrm{Nm}$ for the initial topology. Hence, the designs found using the uncoupled algorithms are still structurally superior to the initial topology; however, because they have been designed for a different load case, they are not as efficient for the real loads acting on the structure as they are for the initial loads. In the next section the coupled solution approach is presented, where the flow is updated with the changing topology.

\subsection{Fluid-structure coupling}

Here the coupled solution is presented. This means an extra parameter, $n_{L B M}$, is defined, which determines when the LBM is solved to update the pressure loads on the structure. In this study two separate analyses are performed: one to compare the suitability of the optimisation algorithms (Sect. 4) and the other to determine the effect that the degree of coupling, or $n_{L B M}$, has on the final design (Sect. 5.1). Therefore, this section is split into three parts, one for each topology optimisation algorithm, with each part performing three analyses with varying degrees of coupling, defined as: weakly coupled $\left(n_{L B M}=10\right)$, strongly coupled $\left(n_{L B M}=5\right)$ and fully cou-

pled $\left(n_{L B M}=1\right)$. These values have been chosen to ensure that the effect of the fluid updates is included in the convergence of the topology optimisation algorithm. Therefore, since the convergence of the algorithm is determined 
by the change in the objective function over the past 10 optimisation iterations, $n_{L B M} \leq 10$ for the fluid-structure coupling to be considered in the convergence criteria.

\subsubsection{Evolutionary algorithm}

First, a BESO algorithm (Sect. 4.1) is implemented with the different degrees of fluid-structure coupling present (Sect. 5.1). Thus, the pressure loads on the structure are updated by performing the LBM with the updated topology every $n_{L B M}$ iterations. Therefore, extra complexities, which will carry a computational burden, are involved in this analysis compared to the uncoupled solutions (Sect. 6.2). However, by modelling the fluid-structure interactions, a more realistic implementation of the physics of the problem is considered, and so the final topology should be superior to the uncoupled result. This has been demonstrated in previous studies that considered design-dependent pressure loads in their analyses (Du and Olhoff (2004a); Picelli et al. (2015a); Munk et al. (2016)).

The initial structure is identical to that used for the uncoupled analysis (Fig. 3c). The optimisation parameters (evolutionary ratio, $E R=0.02$, volume constraint, $V=0.58$, maximum addition ratio, $A R_{\max }=0.02$, and tolerance, $\delta=0.001$ ) are defined before the BESO algorithm (Sect. 4.1) is applied. To determine the effect of the degree of fluid-structure coupling, three different LBM iteration values are imposed by setting $n_{L B M}=10,5$ and 1.

For all three coupled cases (Fig. 9), the final topology is a strikingly different design compared to the uncoupled case. However, comparing the three coupled cases reveals much subtler differences in the designs. The solution is, thus, very sensitive to the fluid-structure interactions, but the degree of coupling does not have much of an effect on the solution so long as it is considered in the convergence criteria of the algorithm (i.e. $n_{L B M} \leq$ 10). The small holes present in the uncoupled solution (Fig. 9a) have been replaced by two larger holes along the x-axis. Moreover, the shapes of the holes have become less triangular, more rounded and rectangular, and the structural members are much simpler. The strain energy distributions for the uncoupled and coupled BESO topologies are given in Fig. 10.

The strain energy density of the uncoupled topology is significantly higher compared to the coupled topologies. The regions of concentrated strain energy in the uncoupled topology are more severe, having a larger difference compared to the rest of the material. The compliances of the datum and 


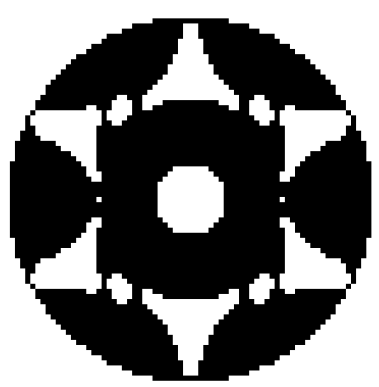

(a) Uncoupled

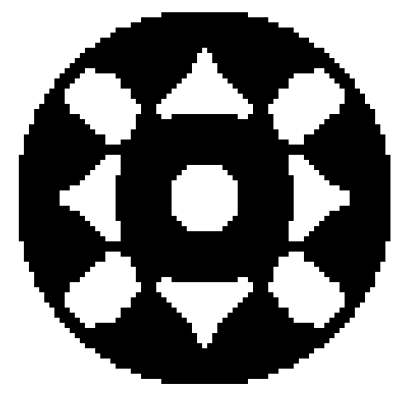

(c) $n_{L B M}=5$

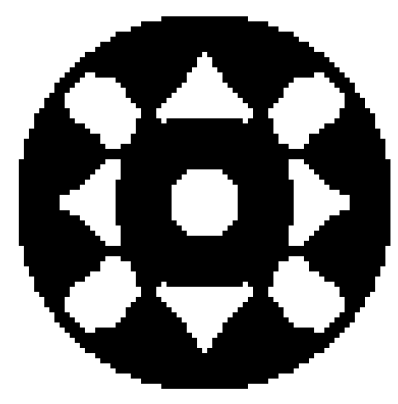

(b) $n_{L B M}=10$

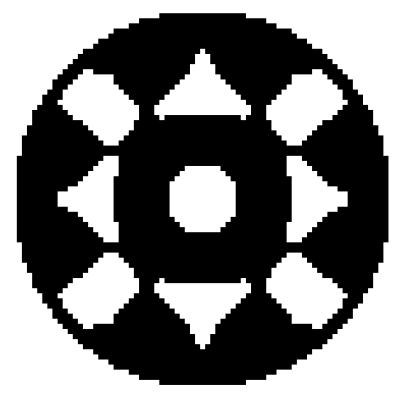

(d) $n_{L B M}=1$

Figure 9: Final BESO-found topologies for the baffle with design-dependent loading.

uncoupled topologies are $5.13 \times 10^{9} \mathrm{Nm}$ and $4.39 \times 10^{9} \mathrm{Nm}$, respectively (Sect. 6.2), whereas, for the coupled topologies, the compliance is further reduced to $2.396 \times 10^{9}, 2.383 \times 10^{9}$ and $2.281 \times 10^{9} \mathrm{Nm}$ for $n_{L B M}=10,5$ and 1 , respectively. Thus, the coupled solutions produce final topologies that use the material of the baffle much more efficiently. The optimiser with the fluid-structure coupling is able to improve the design of the baffle further, reducing the compliance and energy concentrations in the structure.

It is important to note that, as the degree of coupling is increased, i.e. as $n_{L B M}$ is reduced, the structural symmetry, and hence fluid loading about the $\mathrm{x}$ - and $\mathrm{y}$-axes, becomes identical (Fig. 10). The uncoupled solution is symmetric about both the x- and y-axes (Fig. 10a), but not in the same way, i.e. if the topology is rotated $90^{\circ}$ it does not remain the same. This is because the initial structure, and hence the loading, is not symmetric about the x-axis 


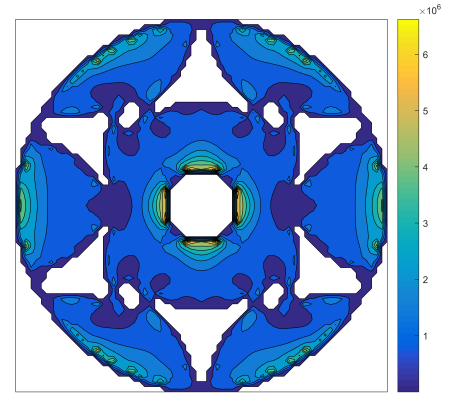

(a) Uncoupled

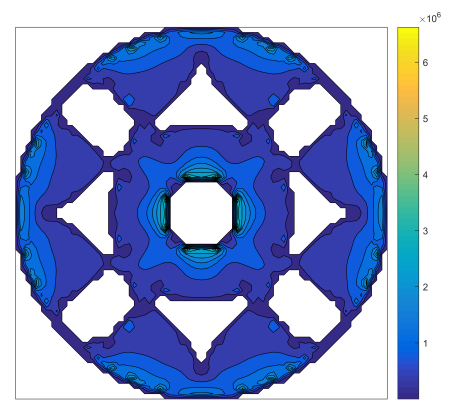

(c) $n_{L B M}=5$

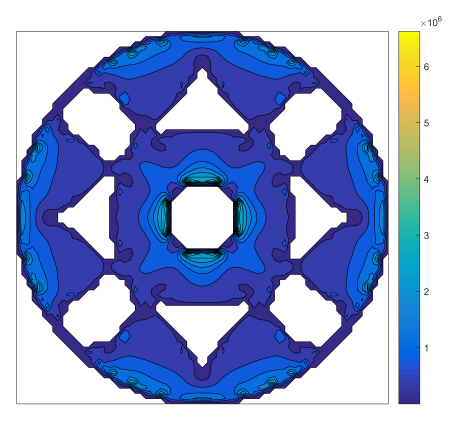

(b) $n_{L B M}=10$

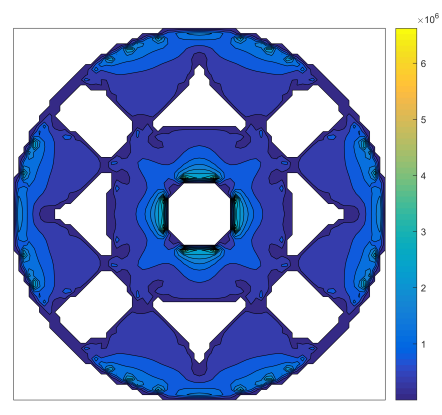

(d) $n_{L B M}=1$

Figure 10: Strain energy distributions for the baffle with design-dependent loading for BESO-found designs.

in the same way as it is about the y-axis. Therefore, because the loading is not updated, this difference in the symmetry remains present for the entire analysis. Thus, the final topology remains symmetrical about both the $\mathrm{x}-$ and y-axes. This is known as 2-fold rotational symmetry, $\mathrm{SO}(2)$, about the centre point. In contrast, the coupled solutions update the fluid mechanics, and hence the pressure loading on the structure. Since there is no physical difference about the horizontal and vertical axes, there is nothing present to introduce asymmetry into the topology. Therefore, it makes physical sense for the fully coupled solution, $n_{L B M}=1$, to have 4 -fold rotational symmetry, $\mathrm{SO}(4)$, about the centre point. Hence, from a physical standpoint, the coupled topologies are shown to be optimal.

The convergence histories for the different degrees of coupling are given in Fig. 11. Due to the fluid-structure coupling, step drops and jumps in the 


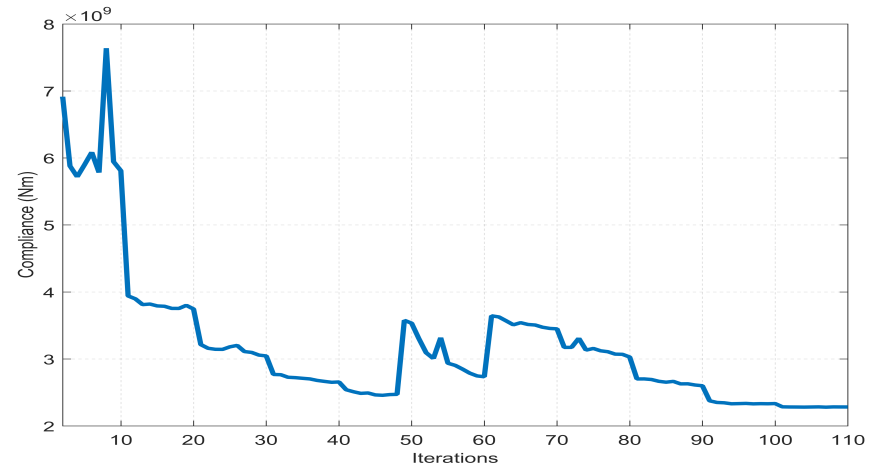

(a) $n_{L B M}=10$

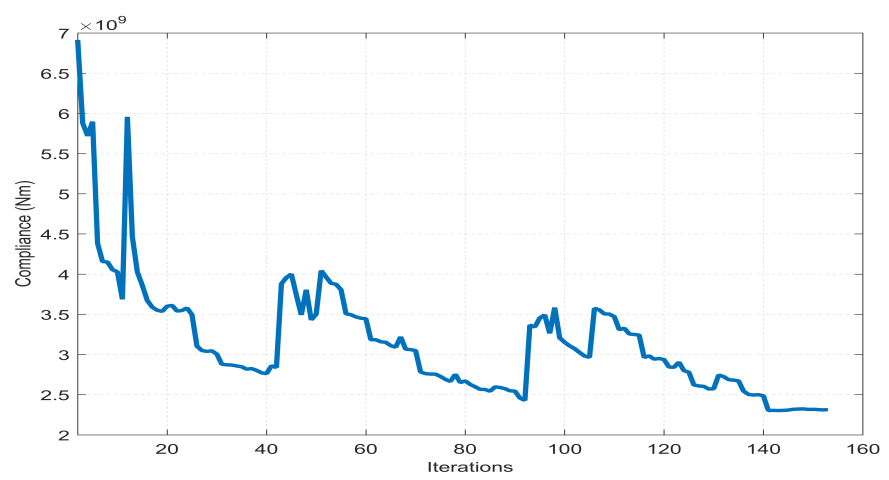

(b) $n_{L B M}=5$

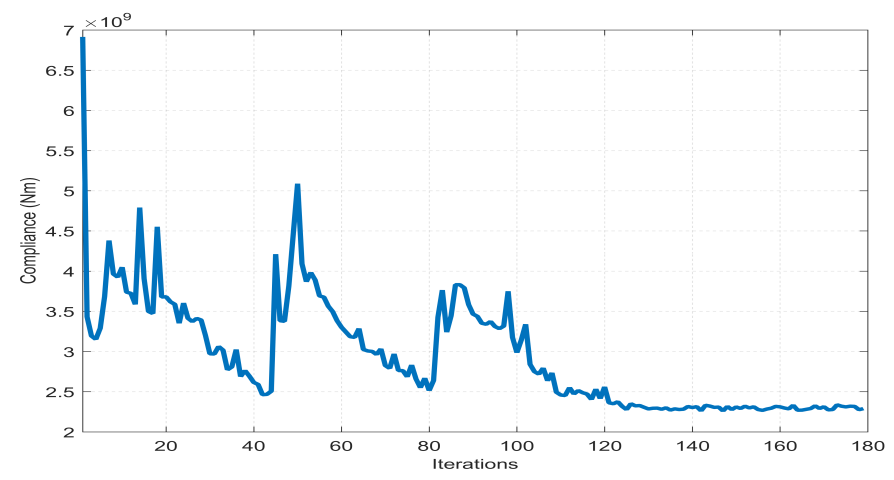

(c) $n_{L B M}=1$

Figure 11: Convergence histories for the baffle with design-dependent loading using BESO. 
objective are observed when the updated topology is run back through the fluid solver. It is found that the step changes in the objective are larger for lower degrees of coupling, hence higher values of $n_{L B M}$, as the pressure loading is updated less often, resulting in a topology that is designed for an inaccurate load case. Thus, although there are more sudden changes in the objective for lower $n_{L B M}$ values, their magnitude is lower. The sequence takes 110, 153 and 179 iterations to converge for the $n_{L B M}=10,5$ and 1 cases, respectively. Hence, it appears that for higher degrees of coupling more iterations are required for convergence. This is because of the convergence criterion for the BESO algorithm (Sect. 4.1). The lower the value of $n_{L B M}$, the smaller the interval between LBM runs, and hence updating of the loads. Since the convergence criterion assesses the change in the objective in the previous 10 iterations, a minimum of 1,2 and 10 LBM runs are performed for the convergence of the $n_{L B M}=10,5$ and 1 cases, respectively. This results in earlier convergence for higher $n_{L B M}$ values.

It is important to note that once the fluid dynamics converge, so too do the structural dynamics of the system. This is seen in the convergence histories (Fig. 11) by observing that, as the sequence progresses, the jumps in the objective are reduced. This is most clearly seen in Fig. 11c, where, after iteration 120, the history flattens out and the update runs, through the LBM, produce very small differences in the objective. This explains why the results are comparatively similar for all the coupled cases, as both the fluid and structural domains converge, resulting in a similar final loading and hence topology. This is a significant benefit of the proposed algorithm, since increasing the degree of coupling results in small improvements in the objective, albeit at a large computational penalty. Therefore, lower degrees of coupling can be implemented with only small sacrifices in the quality of the objective and with large improvements in computational efficiency, which becomes significant when several design architectures are being considered at the conceptual design stage. The results for the different degrees of coupling are summarised in Table 2.

The initial structure has a compliance of $5.13 \times 10^{9} \mathrm{Nm}$, so this is reduced by approximately $53 \%, 54 \%$ and $56 \%$ for $n_{L B M}=10,5$ and 1 , respectively. The main computational burden comes from the LBM runs, since one LBM run takes approximately $3000 \mathrm{CPU}(\mathrm{s})$, compared with $8 \mathrm{CPU}(\mathrm{s})$ for the optimisation routine per optimisation run. Therefore, Table 2 shows a semielliptical growth in the number of LBM runs required for an increase in the degree of coupling. The computational efficiency is reduced by approximately 
Table 2: Summary of results for coupled BESO with multiple degrees of coupling.

\begin{tabular}{rrrr}
$n_{L B M}$ & Minimum compliance $(\mathrm{Nm})$ & Iterations & Number of LBM runs \\
\hline 10 & $2.396 \times 10^{9}$ & 110 & 11 \\
5 & $2.383 \times 10^{9}$ & 153 & 30 \\
1 & $2.281 \times 10^{9}$ & 179 & 179
\end{tabular}

$173 \%, 497 \%$ and $1527 \%$ for $n_{L B M}=10 \rightarrow 5,5 \rightarrow 1$ and $10 \rightarrow 1$, respectively.

Lastly, it is important to investigate how weak the fluid-structure coupling can be made while still achieving near optimal designs. It has been shown that so long as $n_{L B M} \leq 10$ the optimal design is not sensitive to the frequency of updates through the fluid solver. However, this limit ensures that the coupling is included in the convergence criteria. Therefore, to demonstrate that $n_{L B M}$ should remain at 10 or less the problem is now solved with a $n_{L B M}=20$. The final design is illustrated in Fig. 12 .

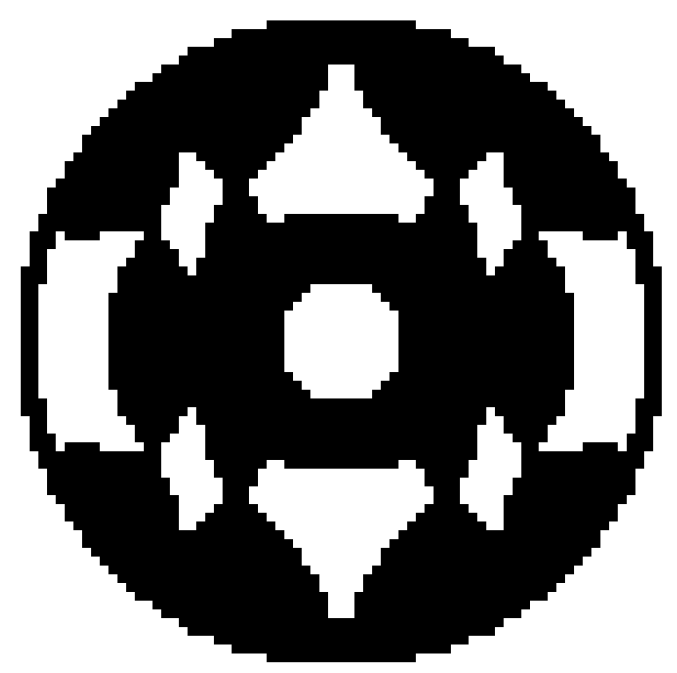

Figure 12: Final topology for the baffle with $n_{L B M}=20$.

Clearly there is a noticeable difference in the final topology produced when $n_{L B M}=20$ (Fig. 12) compared with the other coupled designs (Fig. 9). It is observed that this looser coupling has produced a topology in-between those of the uncoupled and coupled designs, indicating that the coupling needs to be tightened for a better optimum to be found. This is confirmed by the final compliance of the structure shown in Fig. 12 which is $3.3054 \times 10^{9}$ 
$\mathrm{Nm}$, a value almost directly in-between the uncoupled $\left(4.39 \times 10^{9} \mathrm{Nm}\right)$ and weakly coupled, i.e. $n_{L B M}=10,\left(2.396 \times 10^{9} \mathrm{Nm}\right)$ designs. Therefore, it is concluded that the coupling frequency is limited to $n_{L B M} \leq 10$ such that a near optimum is achieved.

\subsubsection{Level-set algorithm}

As shown in Sect. 6.2, the LSM optimisation parameters that produced the most favourable results for the uncoupled case are $\varphi=10^{-5}$ and $n_{v o l}=$ 50. These parameters are used here for all the coupled cases. To examine the effect of the number of LBM iterations, three different degrees of coupling are imposed by setting $n_{L B M}=10,5$ and 1 .

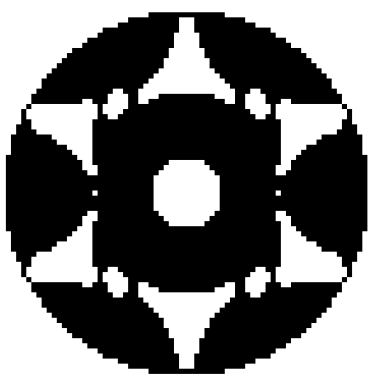

(a) Uncoupled

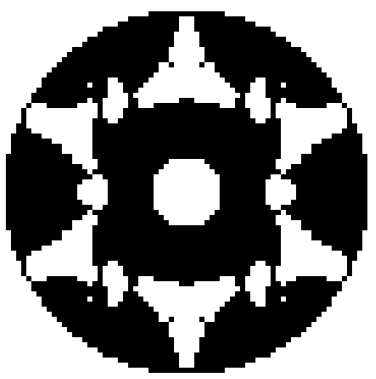

(c) $n_{L B M}=5$

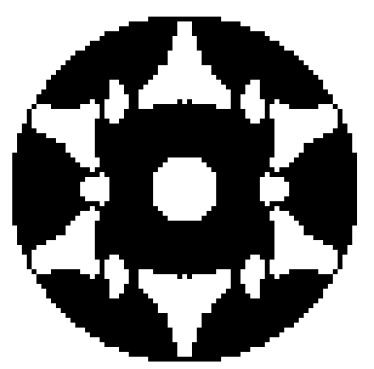

(b) $n_{L B M}=10$

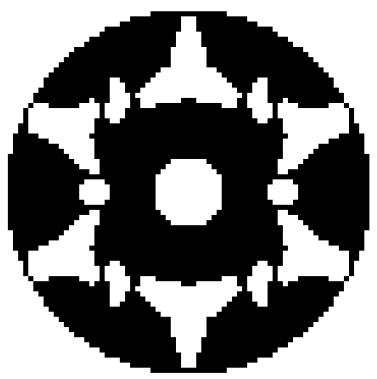

(d) $n_{L B M}=1$

Figure 13: Final LSM-found topologies for the baffle with design-dependent loading.

Similarly to BESO, the final topologies for all the coupled cases (Fig. 13) are comparatively different compared with the uncoupled topology (Fig. 13a). 
However, the coupled topology optimisations have again produced similar final designs, with only subtle differences. The LSM algorithm (Sect. 4.2) is also sensitive to the fluid-structure coupling, although the degree of coupling has a less notable effect on the final topology provided $n_{L B M} \leq 10$. Again, larger holes along the $\mathrm{x}$-axis have replaced the smaller holes from the uncoupled solution. The holes have become more rounded, to further reduce the strain energy concentration. The strain energy distributions for the uncoupled and coupled LSM topologies are given in Fig. 14.

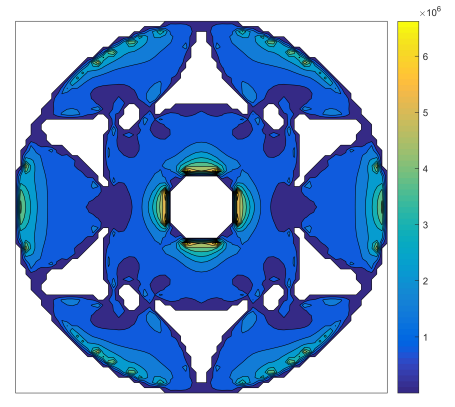

(a) Uncoupled

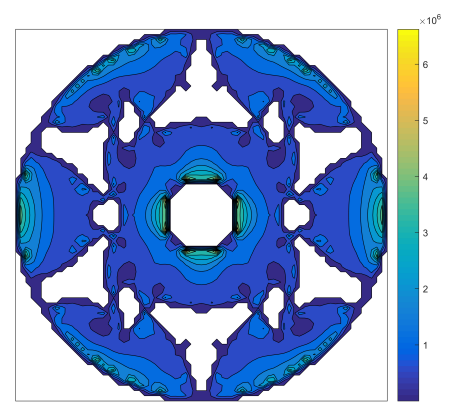

(c) $n_{L B M}=5$

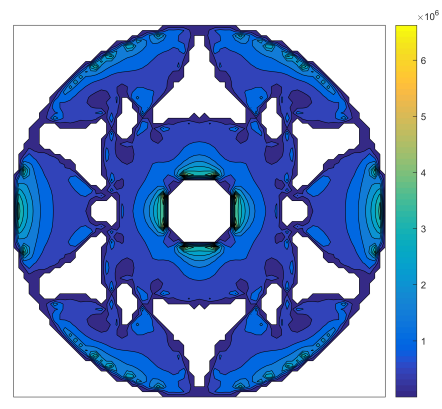

(b) $n_{L B M}=10$

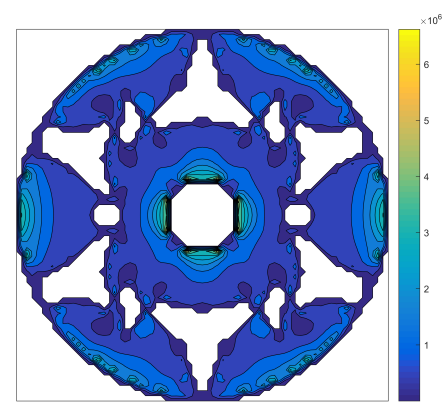

(d) $n_{L B M}=1$

Figure 14: Strain energy distributions for the baffle with design-dependent loading for LSM-found designs.

In a similar fashion to BESO (Fig. 10), the strain energy density of the coupled LSM topologies is significantly reduced compared with the uncoupled topology. Again, the uncoupled topology has larger concentrations of strain energy density. Recall that for the uncoupled topology the final compliance is $4.36 \times 10^{9} \mathrm{Nm}$ (Table 1). However, the final compliance for the coupled 
topologies is further reduced to $3.172 \times 10^{9}, 3.125 \times 10^{9}$ and $3.010 \times 10^{9}$ $\mathrm{Nm}$ for the $n_{L B M}=10,5$ and 1 cases, respectively. Therefore, applying a coupled LSM algorithm produces a more efficient final topology. However, compared to the topologies found by the coupled BESO algorithm, the final LSM topologies are less optimal, since the final compliance is comparatively larger for all coupled cases. Unlike the coupled BESO topologies, the coupled LSM topologies do not approach a $\mathrm{SO}(4)$ design, but remain $\mathrm{SO}(2)$. It was shown in Sect. 6.3.1 that the true optimal design for this test case should be $\mathrm{SO}(4)$, since there is no physical driver of asymmetry, which would cause a difference about the horizontal and vertical axes. Therefore, it can be concluded that, while the coupled LSM algorithm does improve the design of the baffle, compared with the initial and uncoupled designs, it does not approach a global optimum.

This deficiency in the LSM algorithm is likely due to its immaturity as a method. As discussed in Sect. 2, level-set methods are traditionally used for shape optimisation. The algorithm implemented in this work (Sect. 4.2) was only developed recently (Yamada et al. (2010)). It has therefore only been applied to relatively simple topology optimisation problems. Moreover, certain improvements seen in the BESO (Sect. 4.1) and SIMP (Sect. 4.3) algorithms, such as mesh-independency filters (Sigmund and Petersson (1998)) and objective history averaging for convergent results (Huang and Xie (2007)), have not been implemented in the LSM algorithm. Thus, for simple non-designdependent pressure-loaded optimisation problems the algorithms produce comparable results (Sect. 6.2); however, for complex design-dependent coupled problems the LSM is less effective for the type of problem and LSM implementation investigated here.

The convergence histories for the different degrees of coupling are given in Fig. 15. Once more, step drops and jumps in the objective are observed when the updated topology is run back through the fluid solver. Again, these step changes have a larger magnitude for lower degrees of coupling, due to the larger relative change in the topology between LBM runs. Similarly, there are more step changes, of smaller magnitude, for lower $n_{L B M}$ values. Nevertheless, the sequence takes 200, 167 and 189 iterations to converge for the $n_{L B M}=10,5$ and 1 cases, respectively. Therefore, in this case, a higher degree of coupling does not necessarily correspond with more optimisation iterations. However, due to the large difference in $n_{L B M}$ values between the three cases, higher degrees of coupling do result in an increase in the number of LBM runs, and hence in computational cost. 


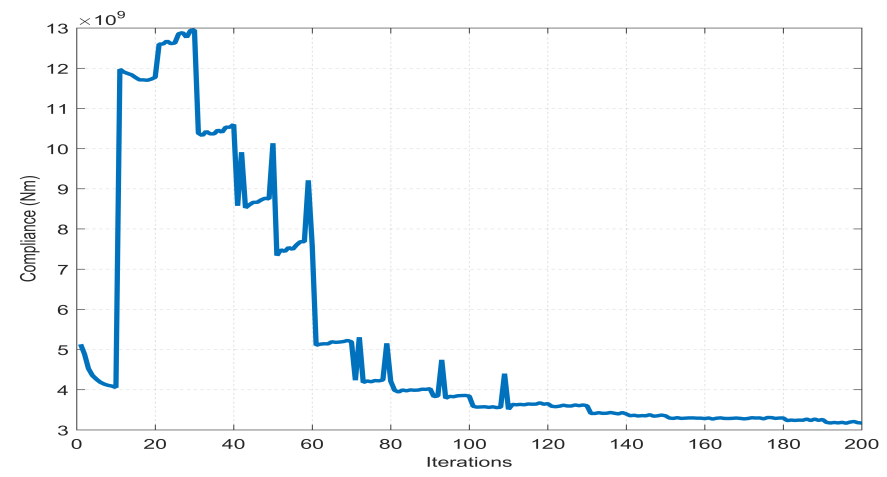

(a) $n_{L B M}=10$

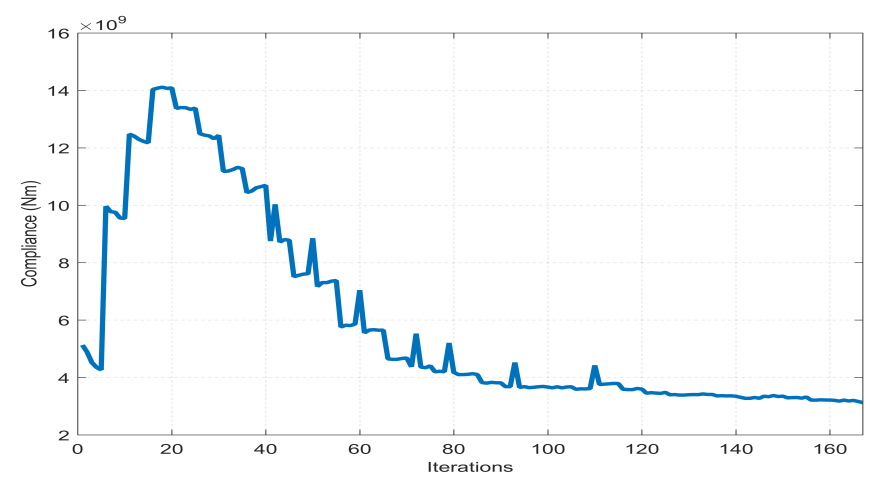

(b) $n_{L B M}=5$

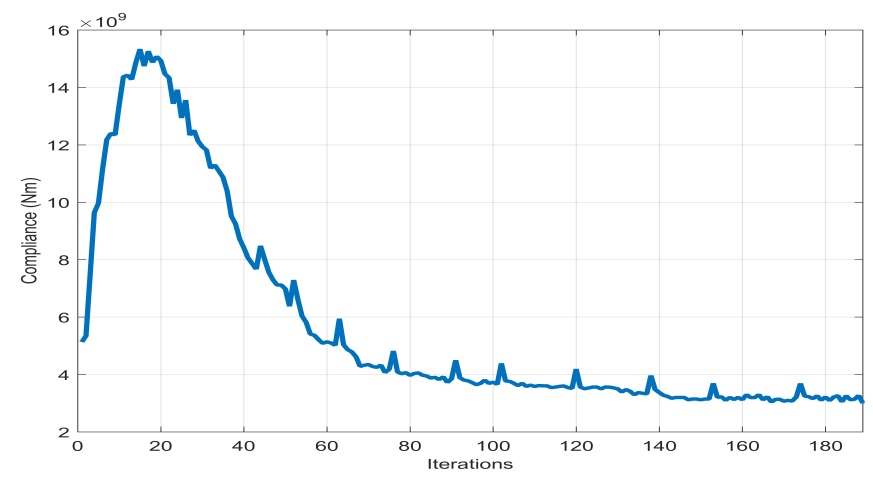

(c) $n_{L B M}=1$

Figure 15: Convergence histories for the baffle with design-dependent loading using LSM. 
Similarly for this analysis, the convergence histories (Fig. 15) show that the fluid and structural dynamics of the system converge together. This is clearly observed by noting that, as the sequence progresses, the jumps in the objective reduce. For all cases, the iteration histories have two distinct regions. The first region shows gradual jumps in the objective followed by gradual drops, as the fluid mechanics and structures begin to converge. The second region is characterised by a smoothing out of the objective, as the solution converges, and the fluid mechanics updates have little effect on the objective. In Sect. 6.3.1 this was shown to be the reason why the coupled final topologies are similar, since both the fluid and structural domains converge. This is again observed here. By comparing the convergence histories of the BESO (Fig. 11) and LSM (Fig. 15) algorithms, it is noted that, for the BESO algorithm, there are several convergence patterns, where the compliance is gradually reduced and then jumps back up, each one reaching a lower compliance than the last, until finally convergence is achieved. In contrast, for the LSM, there is only one convergence, after the initial increase, to a minimum compliance. These convergence patterns suggest that the BESO algorithm is initially going towards local optima, before finally finding a global or nearglobal solution, whereas, conversely, the LSM algorithm likely converges to a local optimum.

The benefit of only small penalties in the objective, with a substantial increase in the computational efficiency, resulting from solutions with a lower degree of coupling, is also present in the LSM cases. As mentioned in Sect. 6.3.1, this becomes a significant advantage in the conceptual design stage when several candidates are being considered. The results for the different degrees of coupling are summarised in Table 3.

Table 3: Summary of results for coupled LSM with multiple degrees of coupling.

\begin{tabular}{rrrr}
\hline$n_{L B M}$ & Minimum compliance $(\mathrm{Nm})$ & Iterations & Number of LBM runs \\
\hline 10 & $3.172 \times 10^{9}$ & 200 & 20 \\
5 & $3.125 \times 10^{9}$ & 167 & 33 \\
1 & $3.010 \times 10^{9}$ & 189 & 189
\end{tabular}

The initial structure has a compliance of $5.13 \times 10^{9} \mathrm{Nm}$, so this is reduced by approximately $38 \%, 39 \%$ and $41 \%$ for the $n_{L B M}=10,5$ and 1 cases, respectively. However, it is again of note that the number of LBM runs is substantially increased, especially for a fully coupled solution (Table 3). 
Hence, the computational efficiency is reduced by approximately $65 \%, 473 \%$ and $845 \%$ for $n_{L B M}=10 \rightarrow 5,5 \rightarrow 1$ and $10 \rightarrow 1$, respectively.

\subsubsection{Solid Isotropic Material with Penalisation}

Finally, the SIMP algorithm (Sect. 4.3) is implemented with the different degrees of fluid-structure coupling present (Sect. 5.1). As outlined in Sect. 2, an extra complexity is present, as the structural boundaries are not clearly defined due to the SIMP algorithm being continuous. The boundaries are therefore approximated using the filter method described in Sect. 5.3. This is expected to have some effect on the final topologies produced by the algorithm, especially when compared with the discrete methods, which do not have this additional complexity.

The optimisation parameters (move limit, $m=0.2$, minimum design variable, $x_{\min }=10^{-3}$, filter radius, $r_{\min }=3$, and tolerance, $\delta=0.01$ ) are defined before the SIMP algorithm (Sect. 4.3) is applied. The uncoupled and coupled topologies found using the SIMP algorithm are given in Fig. 16.

Once again, for all three coupled cases, the final topologies are distinctly different to the uncoupled topology (Fig. 16). However, there is only a small difference between the three coupled designs; this was also evident in topologies given by the BESO (Sect. 6.3.1) and LSM (Sect. 6.3.2) algorithms. Thus, for all topology optimisation methods, the solution is very sensitive to the fluid-structure coupling, but not to the degree of coupling so long as $n_{L B M} \leq 10$. The final coupled topologies for the SIMP algorithm contain a small amount of grey material, since the algorithm is continuous. This is not possible for the discrete BESO and LSM algorithms. For all cases, the small holes in the uncoupled topology (Fig. 16a) are replaced by two larger holes along the x-axis. It is clear that when a coupled solution is implemented the holes that are formed become more rounded (Figs. 9, 13 and 16), with less sharp edges to reduce strain energy concentration. The strain energy distributions for the coupled and uncoupled SIMP topologies are shown in Fig. 17.

For all topology optimisation algorithms used in this work (Sect. 4), the strain energy densities of the uncoupled topologies are significantly higher than the coupled topologies (Figs. 10, 14 and 17). The concentrated regions of strain energy are reduced considerably, in the coupled topologies. For the SIMP algorithm, the final compliance for the uncoupled topology is $4.33 \times$ $10^{9} \mathrm{Nm}$ (Sect. 6.2). In comparison, for the coupled SIMP topologies, the compliance is further reduced to $2.556 \times 10^{9}, 2.558 \times 10^{9}$ and $2.556 \times 10^{9} \mathrm{Nm}$ 


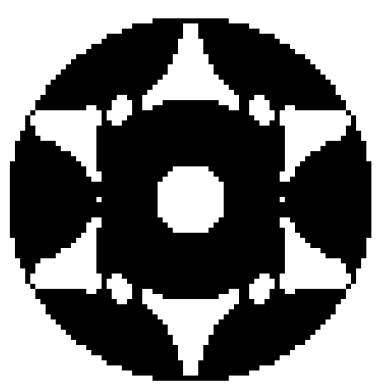

(a) Uncoupled

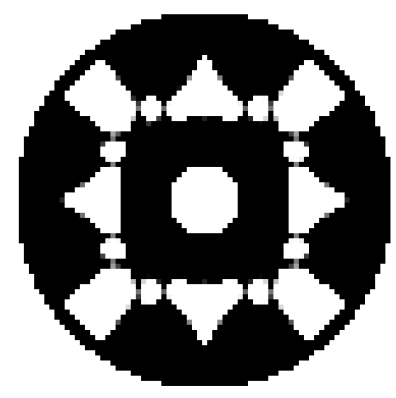

(c) $n_{L B M}=5$

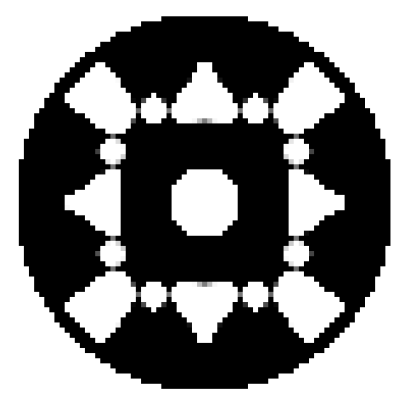

(b) $n_{L B M}=10$

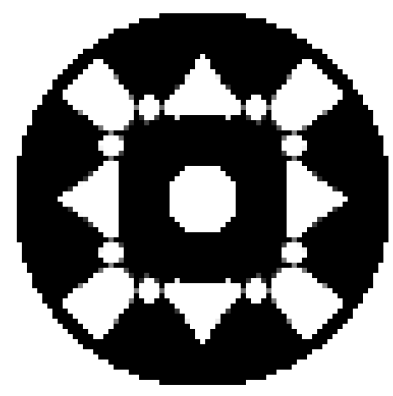

(d) $n_{L B M}=1$

Figure 16: Final SIMP-found topologies for the baffle with design-dependent loading.

for $n_{L B M}=10,5$ and 1 , respectively. Thus, the coupled SIMP algorithm, like the coupled BESO and LSM algorithms, produces more efficient final topologies, reducing the compliance and stress concentrations in the baffle.

Interestingly, by comparing Figs. 10, 14 and 17, it is found that the coupled SIMP topologies represent intermediate solutions to those of the BESO and LSM algorithms, having characteristics of the solutions found by both. The explanation of this similarity comes from two realisations. Firstly, the BESO algorithm is a discrete version of the SIMP algorithm, with a comparable sensitivity analysis and filter scheme, thus the designs are driven to a similar final topology. Secondly, the SIMP algorithm requires an additional method to determine the change in the loaded surface (Sect. 5.3), which the BESO method does not, and, in this case, the method makes use of isolines similar to a level-set parameterisation. Thus, we see the effect 


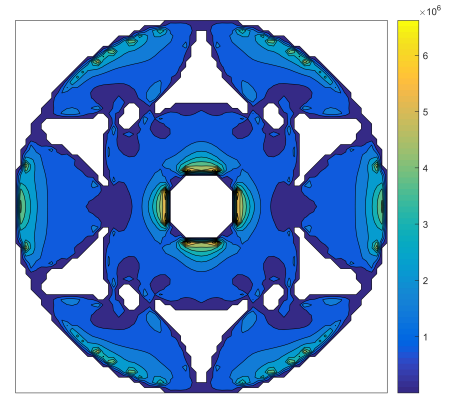

(a) Uncoupled

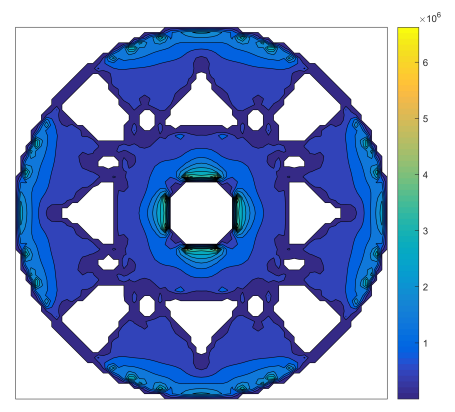

(c) $n_{L B M}=5$

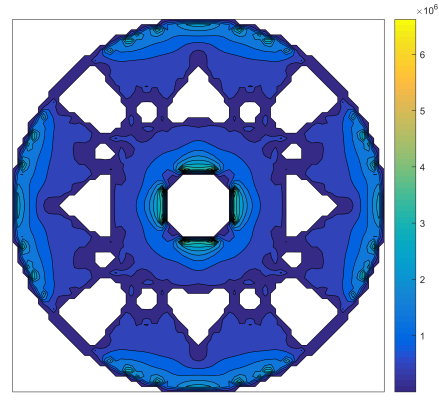

(b) $n_{L B M}=10$

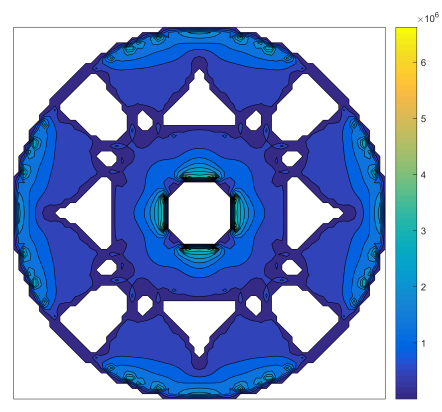

(d) $n_{L B M}=1$

Figure 17: Strain energy distributions for the baffle with design-dependent loading for SIMP-found designs.

the filter (Sect. 5.3) has on the final design. The compliance values for the coupled SIMP topologies are greater than those for the coupled BESO topologies and less than those for the coupled LSM topologies. Thus, the BESO algorithm appears to produce structures closest to the global optimum. As with the BESO algorithm, as the degree of coupling is increased for the SIMP method, the structural symmetry and fluid loading about the $\mathrm{x}$ and $\mathrm{y}$ axes become identical (Fig. 17). So, unlike the LSM, the fully coupled SIMP solution becomes $\mathrm{SO}(4)$, which was shown to be a requirement for optimality in Sect. 6.3.1.

Although it is likely that the filter scheme (Sect. 4.3) has an effect on the results for the coupled SIMP topologies, this effect is minimal. The final compliance values are comparable to those of the BESO algorithm and a significant improvement on the LSM results. The final SIMP topologies 
exhibit 4-fold rotational symmetry and show likeness to the BESO and LSM topologies. Therefore, the filter scheme derived in Sect. 5.3 can be used to apply continuous algorithms to problems with design-dependent pressure loads, similarly to those seen in the literature (Hammer and Olhoff (2000); Du and Olhoff (2004b); Lee and Martins (2012)).

The SIMP convergence histories for the different degrees of coupling are given in Fig. 18. It is clear that step drops and jumps in the objective are present in the convergence histories of all the coupled algorithms (Figs. 11, 15 and 18) when the updated topology is run back through the LBM solver. These step changes have a larger magnitude for lower degrees of coupling. However, only for the coupled SIMP algorithm does the objective drop below the final, converged, value. This occurs because the SIMP algorithm is continuous, and hence in the early iterations the topologies contain a lot of intermediate material. Due to their discrete natures, this does not occur in the BESO and LSM algorithms. As the SIMP optimisation progresses, the intermediate material is penalised and the final structure goes towards a solid-void topology, resulting in a slight increase in the objective.

The convergence histories for the SIMP algorithm (Fig. 18) are seen to be more steady compared with the BESO (Fig. 11) and LSM (Fig. 15) histories. This is because the sensitivity analysis (Sect. 5.2) is based on the idea of an infinitesimal change in the design variables, $\mathbf{x}$, with respect to the objective $C$. Hence, due to the discrete natures of the BESO and LSM algorithms, the sensitivity analysis can become inaccurate (Munk et al. (2017b)), resulting in an unsteady convergence. The SIMP sequences take 154, 203 and 171 iterations to converge for the $n_{L B M}=10,5$ and 1 cases, respectively. Therefore, as for the LSM, higher degrees of coupling do not directly correspond to more optimisation iterations, but do correspond to an increase in the number of LBM runs, and therefore more computational expense.

For all cases with $n_{L B M} \leq 10$, it is shown that the fluid and structural dynamics both converge for the coupled solutions (Figs. 11, 15 and 18). For the SIMP algorithm, this corresponds to the removal of intermediate material and the formation of a nearly solid-void topology. This is observed in the iteration histories (Fig. 18) by noting that, as the sequence progresses, the jumps in the objective are reduced. After a certain number of iterations the runs through the LBM solver have a minimal effect on the objective, which is why the coupled topologies are similar.

An important benefit, observed in all the topology optimisation algorithms (Sect. 4), is that lower degrees of coupling, up until $n_{L B M}=10$, 


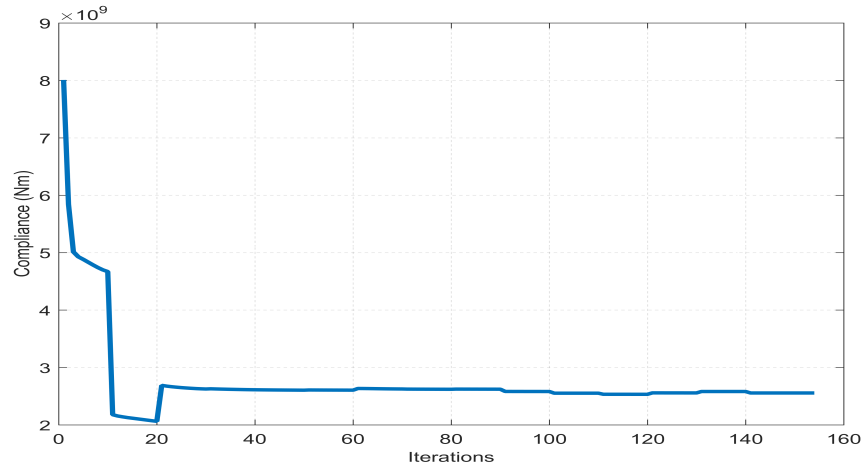

(a) $n_{L B M}=10$

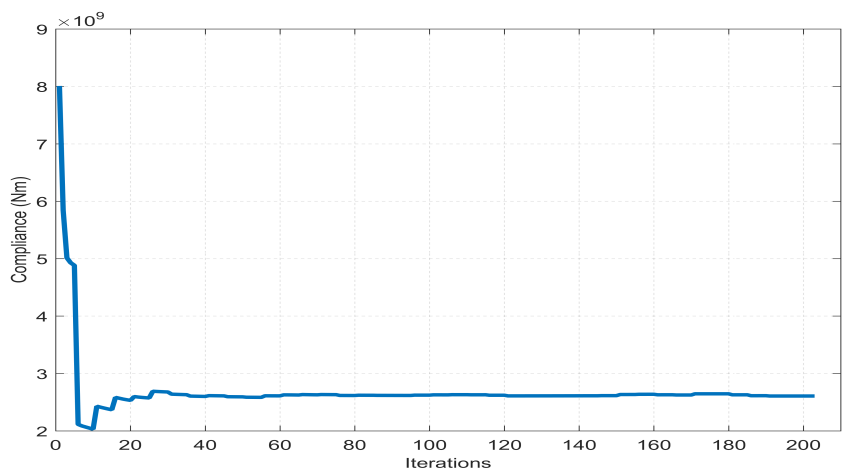

(b) $n_{L B M}=5$

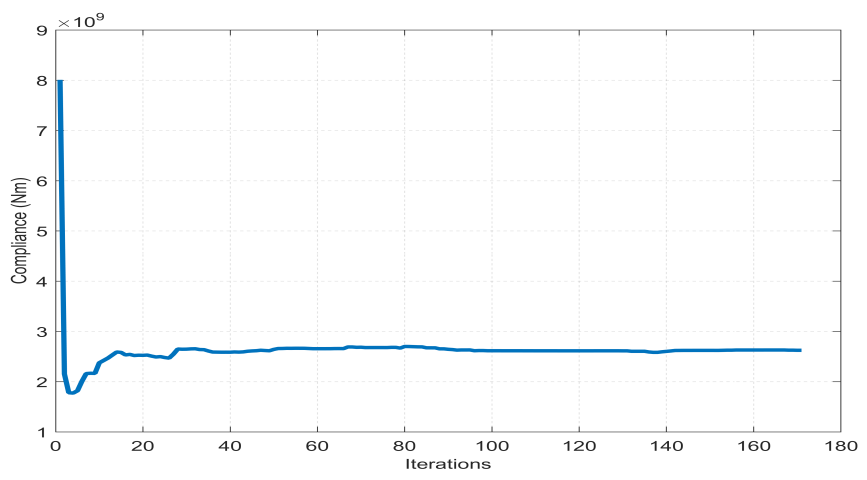

(c) $n_{L B M}=1$

Figure 18: Convergence histories for the baffle with design-dependent loading using SIMP. 
result in small penalties in the objective but a substantial increase in computational efficiency. When several candidate designs are being considered in the early stages of the design process, this benefit becomes significant. The results for the different degrees of coupling are summarised in Table 4.

Table 4: Summary of results for coupled SIMP with multiple degrees of coupling.

\begin{tabular}{rrrr}
\hline$n_{L B M}$ & Minimum compliance $(\mathrm{Nm})$ & Iterations & Number of LBM runs \\
\hline 10 & $2.556 \times 10^{9}$ & 154 & 15 \\
5 & $2.558 \times 10^{9}$ & 203 & 40 \\
1 & $2.556 \times 10^{9}$ & 171 & 171
\end{tabular}

The initial structure has a compliance of $5.13 \times 10^{9} \mathrm{Nm}$; hence, the compliance is reduced by $50 \%$ for all of the coupled SIMP topologies. However, as with the BESO and LSM algorithms, the number of LBM runs is noticeably increased for higher degrees of coupling (Table 4). Therefore, the computational efficiency is reduced by approximately 167\%, 328\% and $1040 \%$ for $n_{L B M}=10 \rightarrow 5,5 \rightarrow 1$ and $10 \rightarrow 1$, respectively.

\subsection{Robustness with regards to the problem parameters}

It has been demonstrated that the coupling algorithm is robust with regards to the optimisation method (Sec. 6.3). Furthermore, the coupling frequency, i.e. how often the LBM is solved, has been shown to have little effect on the final topology so long as $n_{L B M} \leq 10$. However, so far the problem parameters have remained unchanged, i.e. the material properties, $E$, and flow conditions, Re, are fixed. Therefore, in this section the robustness of the coupled optimisation algorithm will be demonstrated by changing the problem parameters and observing the effect they have on the optimiser. Only the BESO algorithm will be used in this analysis, since it was shown to produce the designs with the lowest compliance.

\subsubsection{Effect of material parameters}

The optimisation problem solved in this study has a compliance minimisation objective. Therefore, the only material property that has an effect on the problem is the material stiffness, which is determined by the Young's modulus $E$. Thus, to determine the effect of the stiffness on the optimisation algorithm the same problem is solved with a Young's modulus of $E=0.95 E_{0}$ and $E=1.05 E_{0}$. It must be noted that because the linear problem is solved 
here (Sec. 3.1) it is expected that the stiffness will have a linear effect on the algorithm and therefore only act to scale the objective value (compliance) accordingly. The final designs found using the different stiffness values are illustrated in Fig. 19.

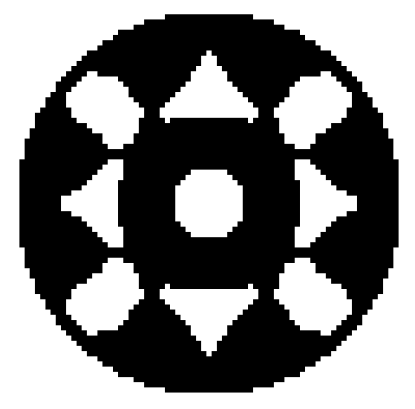

(a) $E=0.95 E_{0}$

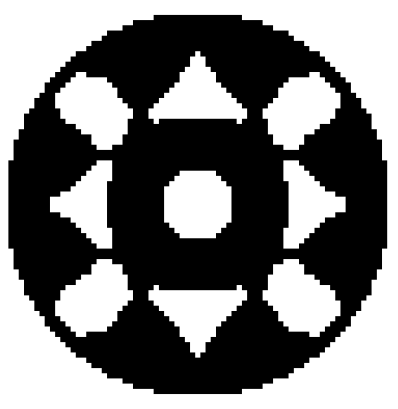

(b) $E=E_{0}$

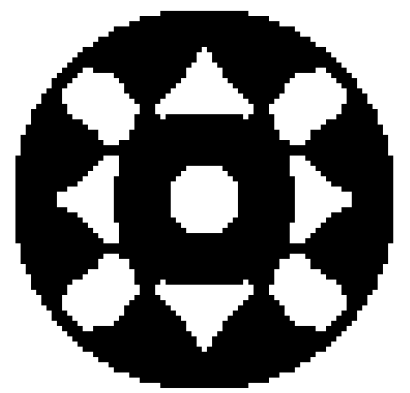

(c) $E=1.05 E_{0}$

Figure 19: Effect of material paramters on final design.

As predicted, it is observed that the final topologies are identical (Fig. 19). The final compliance for the three designs are $2.662 \times 10^{9}, 2.396 \times 10^{9}$ and $1.972 \times 10^{9} \mathrm{Nm}$ for the $E=0.95 E_{0}, E=E_{0}$ and $E=1.05 E_{0}$ cases, respectively. Therefore, the effect of the material parameters is only to scale the compliance, since increasing the Young's modulus increases the stiffness of the structure linearly, and therefore does not affect the solution (i.e. final design) of the algorithm.

\subsubsection{Effect of flow parameters}

Similarly to the material parameters, the robustness of the optimisation algorithm on the flow parameters, determined by the inlet condition set in the LBM, needs to be addressed. The inlet condition for the LBM is the inlet Reynolds number Re (Sec. 3.2). The Reynolds number is the ratio of inertial forces to viscous forces within a fluid. Therefore, altering the Reynolds number essentially changes the physics of the problem, since the flow either becomes more dominated by internal or viscous forces depending which way the Reynolds number is modified. With this in mind, it is expected that, unlike the material parameters, modifying the flow parameters will have a non-linear effect on the optimisation algorithm, thereby changing the final design produced. Furthermore, because the flow parameters are changed, it will be interesting to observe if this has an effect on the required coupling frequency to the flow solver (LBM) to achieve a good optimum. 
The Reynolds number has, thus far, been kept constant at $R e=100$ (Secs. 6.1-6.3). To determine the sensitivity of the algorithm to the Reynolds number, two more problems are defined. The first having a $R e=50$ and the second having a $R e=200$. When $R e=100$ it was shown that if the frequency of the coupling was kept $n_{L B M} \leq 10$ a near optimal solution was found. Therefore, the final topologies found for the three problems with different Reynolds numbers and $n_{L B M}=10$ are shown in Fig. 20.

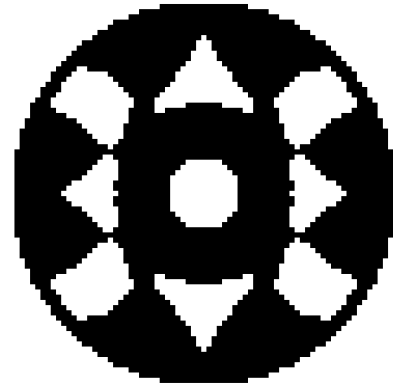

(a) $R e=50$

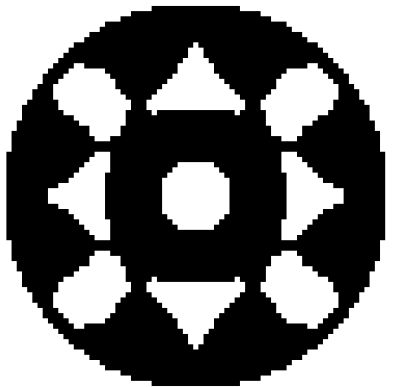

(b) $R e=100$

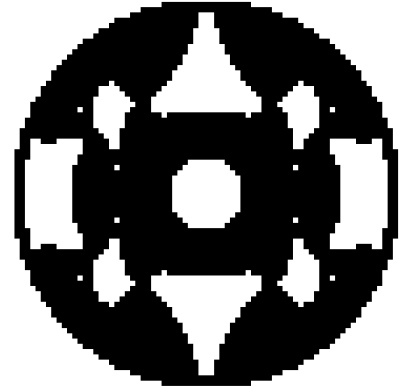

(c) $R e=200$

Figure 20: Effect of flow paramters on final design.

The first observation is that for the $R e=50$ and $R e=100$ cases the final topologies are consistent (Fig. 20). Conversely, the $R e=200$ case produces a noticeably different final topology. The final compliance for the three designs shown in Fig. 20 are $4.2617 \times 10^{8}, 2.396 \times 10^{9}$ and $2.0107 \times 10^{10}$ $\mathrm{Nm}$ for the $R e=50, R e=100$ and $R e=200$ cases, respectively. The fact that the compliance values increase with increasing $R e$ makes physical sense, since a higher Reynolds number translates to a higher pressure difference across the baffle and thus a higher force exerted on the baffle. Nonetheless, the $R e=200$ case appears to have not found a solution near the optimum, unlike the $R e=50$ and $R e=100$ cases. Therefore, this suggests that the fluid-structure coupling needs to be tightened. To validate this conclusion the same three cases are solved, but this time with a tighter coupling parameter $n_{L B M}=5$. The final topologies are given in Fig. 21 .

This time the $R e=200$ case produces a consistent design to the other cases (Fig. 21), confirming our previous hypothesis that higher Reynolds numbers require a tighter fluid-structure coupling to find an optimum solution. This is physically sound since, as previously mentioned, increasing the Reynolds number results in an increase in the force on the baffle and thus, 


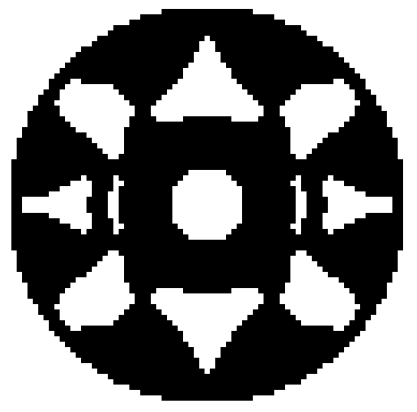

(a) $R e=50$

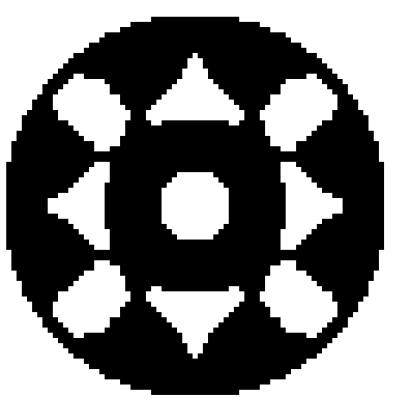

(b) $R e=100$

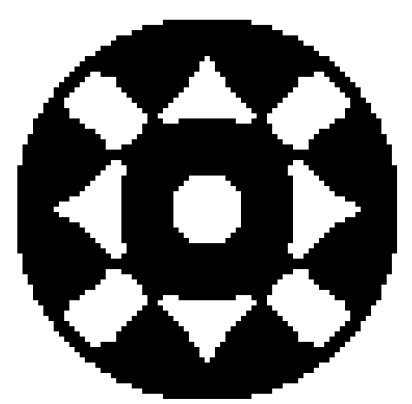

(c) $R e=200$

Figure 21: Effect of flow paramters on coupling frequency.

through eq. 6, a larger dependence of the flow properties on the optimisation algorithm. Therefore, as a result of this larger dependence, the flow properties need to be updated more frequently to ensure the optimiser can find an optimum solution. The final compliance of the three designs given in Fig. 21 are $4.222 \times 10^{8}, 2.383 \times 10^{9}$ and $1.4997 \times 10^{10} \mathrm{Nm}$ for the $R e=50$, $R e=100$ and $R e=200$ cases, respectively. One final observation is that for the $n_{L B M}=10$ cases the relative increase in compliance for the final designs are 5.62 and 8.39 for $R e=50 \rightarrow 100$ and $R e=100 \rightarrow 200$, respectively. However, for the $n_{L B M}=5$ cases the relative increases are 5.64 and 6.29 for $R e=50 \rightarrow 100$ and $R e=100 \rightarrow 200$, respectively. Hence, both the $R e=50$ and $R e=100$ cases have found comparative optimal solutions for both degrees of coupling, since their relative compliances have stayed almost equal. Conversely, the $R e=200$ case found a far superior solution when the coupling was tightened, as evidence of the large reduction in the relative compliance.

\subsection{Summary}

Finally, the results of the structural optimisation algorithms (Sect. 4) are summarised, comparing computational efficiency with the optimality of the final structure. The uncoupled cases (Sect. 6.2) produced the least compliant structures. However, the designs were shown to be improved compared with the initial configuration and loading. Since fluid-structure interactions were not considered, and hence the pressure loading was not updated, this case only required one LBM simulation to obtain the initial loads. All the uncoupled algorithms required fewer iterations for convergence compared with the 
corresponding coupled solutions. Therefore, the uncoupled algorithms represent the most computationally efficient solution possible. This is as expected, since the uncoupled case is the simplest and least accurate; it is predictable that increased non-linearity in the model will increase the number of iterations required to obtain a solution. Thus, if only small improvements are required, towards the end of the design cycle, where changes become costly, the uncoupled algorithms are recommended.

For all cases, the coupled algorithms were able to improve the objective compared to the uncoupled solutions. Increasing the degree of coupling usually increased the optimality of the final structure. However, for all cases, reducing $n_{L B M}$ resulted in a significant increase in the computational expense of the algorithm. It was also observed that, for the BESO and SIMP algorithms, including the fluid-structure coupling resulted in a 4-fold rotationally symmetric structure, which was shown to be physically consistent. Additionally, it was found that, while the solution was sensitive to the fluid-structure interactions, as is evident from the large improvement in the objective obtained for all coupled solutions compared with the uncoupled case, the degree of coupling appears to have only a small impact on the final topology provided $n_{L B M} \leq 10$. Therefore, lower degrees of coupling can be used, with small penalties in the quality of the objective, but large gains in computational efficiency. In addition, the general optimal topology has been identified. Table 5 summarises the structural optimisation results of this work and Fig. 22 provides a graphical representation of the computational burden versus objective value for the different analyses performed.

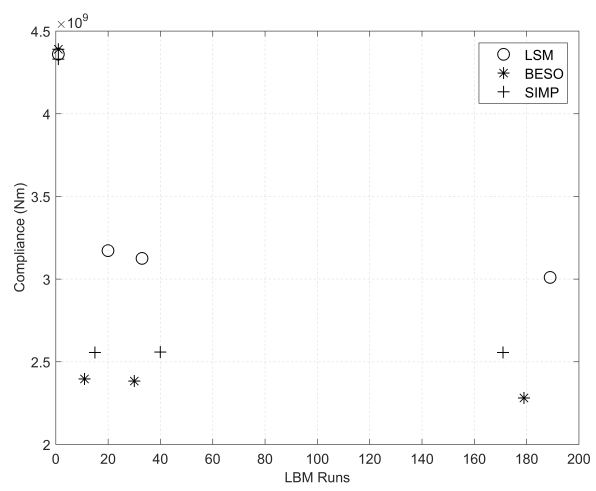

Figure 22: Objective values versus the number of LBM runs for the different algorithms. 
Table 5: Summary of results for structural optimisation.

\begin{tabular}{lllll}
\hline $\begin{array}{l}\text { Optimisation } \\
\text { method }\end{array}$ & $n_{L B M}$ & $\begin{array}{l}\text { Minimum } \\
\text { compliance }(\mathrm{Nm})\end{array}$ & $\begin{array}{l}\text { Iterations } \\
\text { Number of } \\
\text { LSM }\end{array}$ Uncoupled & $\begin{array}{l}4.360 \times 10^{9} \\
\text { LBuns }\end{array}$ \\
LSM & 10 & $3.172 \times 10^{9}$ & 59 & 1 \\
LSM & 5 & $3.125 \times 10^{9}$ & 167 & 200 \\
LSM & 1 & $3.010 \times 10^{9}$ & 189 & 189 \\
BESO & Uncoupled & $4.390 \times 10^{9}$ & 60 & 1 \\
BESO & 10 & $2.396 \times 10^{9}$ & 110 & 11 \\
BESO & 5 & $2.383 \times 10^{9}$ & 153 & 30 \\
BESO & 1 & $2.281 \times 10^{9}$ & 179 & 179 \\
SIMP & Uncoupled & $4.330 \times 10^{9}$ & 133 & 1 \\
SIMP & 10 & $2.556 \times 10^{9}$ & 154 & 15 \\
SIMP & 5 & $2.558 \times 10^{9}$ & 203 & 40 \\
SIMP & 1 & $2.556 \times 10^{9}$ & 171 & 171
\end{tabular}

As mentioned in Sect. 6.3.1, the main computational burden comes from the number of LBM runs required. Table 5 shows a dramatic increase in the number of LBM runs performed in the fully coupled solutions $\left(n_{L B M}=1\right)$. This increase becomes significant when comparing the computational times for each coupled analysis. Table 6 shows the computational cost, for each coupled analysis, on an Intel@ Core $^{\mathrm{TM}}$ i7-2720QM CPU architecture @ 2.20 $\mathrm{GHz}$ when using eight cores in parallel for the LBM simulations.

Table 6: Computational cost for coupled solutions.

\begin{tabular}{llc}
\hline Optimisation method & $n_{L B M}$ & Computational cost (CPU-days) \\
\hline LSM & 10 & 0.7 \\
LSM & 5 & 1.2 \\
LSM & 1 & 6.6 \\
BESO & 10 & 0.4 \\
BESO & 5 & 1.1 \\
BESO & 1 & 6.2 \\
SIMP & 10 & 0.5 \\
SIMP & 5 & 1.4 \\
SIMP & 1 & 6.0
\end{tabular}


Thus, at the conceptual design stage, lower degrees of coupling can be used, to identify approximately optimal designs, with only the final designs being run through a tightly coupled optimisation algorithm.

\section{Conclusions}

A numerical framework for a coupled fluid-structure topology optimisation problem with design-dependent pressure loads has been presented, with the baffle plate of a micro fluidic mixing device being optimised. BESO, LSM and SIMP topology optimisation algorithms have been applied and compared for a minimum compliance objective, with and without the fluid-structure coupling. To the best of the authors' knowledge, this has not yet been seen in the literature of topology optimisation. Previous studies have focused on simplified 2D problems due to the intensive computation required. The application of topology optimisation to structures with design-dependent fluid pressure loads has also been handicapped by the numerous design iterations required, with problem sizes being restricted so that acceptable computation times are achieved (Andreasen et al. (2009)).

An uncoupled analysis was performed with the BESO, LSM and SIMP topology optimisation algorithms to maximise the stiffness of the baffle. This analysis proved to be the most computationally efficient, since only one LBM simulation was required. Moreover, the uncoupled analyses required the fewest iterations to achieve convergence. Nevertheless, the compliance was reduced by $14 \%, 15 \%$ and $16 \%$ for the uncoupled BESO, LSM and SIMP algorithms, respectively. Thus, improvements in the design were achieved without a high computational burden. It was concluded that this analysis would be most beneficial towards the end of the design process when large changes are no longer permitted.

A coupled analysis was also performed with the BESO, LSM and SIMP topology optimisation algorithms, where the pressure loads on the baffle were updated with the change in structural topology. Thus, the fluid-structure interactions were considered in the optimisation of the baffle. The degree of coupling was analysed by changing the number of iterations between LBM runs by setting $n_{L B M}=10,5$ and 1 . It was shown that, for all cases, the coupled algorithms were able to significantly improve the objective compared to the uncoupled solutions. Therefore, it is concluded that the fluid-structure interactions have a notable effect on the optimisation problem. However, it was found that, for each optimisation algorithm, the different coupling lev- 
els produced similar results, with only a small improvement in the objective with greater coupling. This was due to the convergence of both the fluid and structural domains in all the coupled analyses. Thus, the solution is not very sensitive to the degree of coupling provided $n_{L B M} \leq 10$. It was also shown that increasing the degree of coupling resulted in a significant increase in the computational expense of the algorithms. Therefore, lower degrees of coupling can be used, with small penalties on the objective, but at a comparatively lower computational cost. The optimisation process and solution quality can be further improved by initially setting $n_{L B M}=10$ and tightening the constraint by reducing $n_{L B M}$ to 1 once the general optimal topology has been identified. Thus, at the conceptual design phase, where several structural concepts are being considered, this analysis becomes beneficial.

The work presented here brings high-fidelity methods, such as Lattice Boltzmann flow simulations, forward to the conceptual/preliminary design stage. Furthermore, multiple different optimisation algorithms were compared to determine which one was most suitable for the problem being studied. This type of analysis is too infrequently seen in the literature and is imperative for the continued use of topology optimisation in real-world problems. From the results of this study, it is concluded that a medium frequency of fluid mechanics updates on the BESO algorithm produces the best results when physical interpretation, optimality and computational cost are considered. Including an uncertainty analysis to determine robust optima is planned for future work.

\section{Acknowledgements}

D.J. Munk thanks the Australian government for their financial support through the Endeavour Fellowship scheme.

\section{References}

Allaire, G., Gournay, F., Jouve, F., Toader, A.-M., 2005. Structural optimization using topological and shape sensitivity via a level-set method. Control and Cybernetics 34, 59-80.

Allaire, G., Jouve, F., Toader, A.-M., 2004. Structural optimization using sensitivity analysis and a level-set method. Journal of Computational Physics 194, 363-393. 
Amstutz, S., Andra, H., 2006. A new algorithm for topology optimization using a level-set method. Journal of Computational Physics 216, 573-588.

Andreasen, C., Gersborg, A., Sigmund, O., 2009. Topology optimization of microfluidic mixers. International Journal for Numerical Methods in Fluids $61,498-513$.

Andreasen, C., Sigmund, O., 2013. Topology optimization of fluid-structure interaction problems in poroelasticity. Computer Methods in Applied Mechanics and Engineering 258, 55-62.

Bendsøe, M., 1988. Generating optimal topologies in structural design using a homogenization method. Computer Methods in Applied Mechanics and Engineering 71, 197-224.

Bendsøe, M., 1989. Optimal shape design as a material distribution problem. Structural Optimization 1, 193-202.

Bendsøe, M., Sigmund, O., 1999. Material interpolation schemes in topology optimization. Archive of Applied Mechanics 69 (9-10), 635-654.

Bhatnagar, P., Gross, E., Krook, M., 1954. A model for collision prosses in gases. I. small amplitude processes in charged neutral one-component system. Physical Review 94, 511-525.

Borrvall, T., Petersson, J., 2003. Topology optimization of fluids in Stokes flow. International Journal for Numerical Methods in Fluids 41, 77-107.

Bourdin, B., Chambolle, A., 2003. Design-dependent loads in topology optimization. ESAIM: Control, Optimization and Calculus of Variations 9, $19-48$.

Bruggi, M., Cinquini, C., 2009. An alternative truly-mixed formulation to solve pressure load problems in topology optimization. Computer Methods in Applied Mechanics and Engineering 198, 1500-1512.

Burger, M., Hackl, B., Ring, W., 2004. Incorporating topological derivatives into level set methods. Journal of Computational Physics 194, 334-362.

Challis, V., Guest, J., 2009. Level set topology optimization of fluids in Stokes flow. International Jounral for Numerical Methods in Engineering 79, 1284-1308. 
Chandrupatla, T., Belegundu, A., 1997. Introduction to Finite Elements in Engineering, 2nd Edition. Prentice Hall.

Chen, B., Kikuchi, N., 2001. Topology optimization with design-dependent loads. Finite Elements in Analysis and Design 37, 57-70.

D'Ammaro, A., Kipouros, T., Savill, A., Lyazid, D., 2011. Computational design for micro fluidic devices using lattice Boltzmann and heuristic optimisation algorithms. European Numerical Mathematics and Advanced Applications (ENUMATH) Conference, Leicester UK.

Deaton, J., Grandhi, R., 2014. A survey of structural and multidisciplinary continuum topology optimization. Structural and Multidisciplinary Optimization 49, 1-38.

Djenidi, L., Moghtaderi, B., 2006. Numerical investigation of laminar mixing in a coaxial microreactor. Journal of Fluid Mechanics 568, 223-243.

Du, J., Olhoff, N., 2004a. Topological optimization of continuum structures with design-dependent surface loading - part I: New computational approach for 2D problems. Structural and Multidisciplinary Optimization $27,151-165$.

Du, J., Olhoff, N., 2004b. Topological optimization of continuum structures with design-dependent surface loading - part II: Algorithms and examples for 3D problems. Structural and Multidisciplinary Optimization 27, 166177.

Duhring, M., Jensen, J., Sigmund, O., 2008. Acoustic design by topology optimization. Journal of Sound and Vibration 317, 557-575.

Dunning, P. D., Kim, H. A., 2013. A new hole insertion method for level set based structural topology optimization. International Journal for Numerical Methods in Engineering 93, 118-134.

Eschenauer, H., Kobelev, V., Schumacher, A., 1994. Bubble method for topology and shape optimization of structures. Structural Optimization $8,42-51$.

Eschenauer, H., Olhoff, N., 2001. Topology optimization of continuum structures: A review. Applied Mechanics Reviews 54, 331-390. 
Fuchs, M., Moses, E., 2000. Optimal structural topologies with transmissible loads. Structural and Multidisciplinary Optimization 19, 263-273.

Fuchs, M., Shemesh, N., 2004. Density-based topological design of structures subjected to water pressure using a parametric loading surface. Structural and Multidisciplinary Optimization 28 (1), 11-19.

Gao, T., Zhang, W., 2009. Topology optimization of multiphase material structures under design dependent pressure loads. International Journal of Simulation and Multidisciplinary Design Optimization 3 (1), 297-306.

Haber, R., Bendsøe, M., 1998. Problem formulation, solution procedures and geometric modeling - key issues in variable topology optimization. In: Proceedings of the 7th AIAA/USAF/NASA/ISSMO Symposium on Multidisciplinary Analysis and Optimization. AIAA, pp. 1864-1873.

Hammer, V., Olhoff, N., 2000. Topology optimization of continuum structures subjected to pressure loading. Structural and Multidisciplinary Optimization 19, 85-92.

He, L., Kao, C., Osher, S., 2007. Incorporating topological derivatives into shape derivatives based level set methods. Journal of Computational Physics 225, 891-909.

Huang, X., Xie, Y., 2007. Convergent and mesh-independent solutions for the bi-directional evolutionary structural optimization method. Finite Elements in Analysis and Design 43, 1039-1049.

Huang, X., Xie, Y., 2009. Bi-directional evolutionary topology optimization of continuum structures with one or multiple materials. Computational Mechanics 43, 393-401.

Huang, X., Xie, Y., 2010. Evolutionary Topology Optimization of Continuum Structures: Methods and Applications, 1st Edition. John Wiley \& Sons.

Jenkins, N., Maute, K., 2016. An immersed boundary approach for shape and topology optimization of stationary fluid-structure interaction problems. Structural and Multidisciplinary Optimization 54, 1191-1208.

Kreissl, S., Pingen, G., Evgrafov, A., Maute, K., 2010. Topology optimization of flexible micro-fluidic devices. Structural and Multidisciplinary Optimization 42, 495-516. 
Laniewski-Wollk, L., Rokicki, J., 2016. Adjoint lattice Boltzmann for topology optimization on multi-GPU architecture. Computers \& Mathematics with Applications 71 (3), 833-848.

Lee, E., Martins, J., 2012. Structural topology optimization with designdependent pressure loads. Computer Methods in Applied Mechanics and Engineering 233-236, 40-48.

Liu, Z., Korvink, J., Huang, R., 2005. Structure topology optimization: Fully coupled level set method via FEMLAB. Finite Elements in Analysis and Design 29, 407-417.

Makhija, D., Pingen, G., Yang, R., Maute, K., 2012. Topology optimization of multi-component flows using a multi-relaxation time lattice Boltzmann method. Computers and Fluids 67, 104-114.

Mlejnek, H., 1992. Some aspects of the genesis of structures. Structural Optimization 5, 64-69.

Moghtaderi, B., Shames, I., Djenidi, L., 2006. Microfluidic characteristics of a multi-holed baffle plate micro-reactor. International Journal of Heat and Fluid Flow 27, 1069-1077.

Munk, D., Kipouros, T., Vio, G., Parks, G., Steven, G., 2018. Multiobjective and multi-physics topology optimization using an updated smart normal constraint bi-directional evolutionary structural optimization method. Structural and Multidisciplinary Optimization 57 (2), 665-688.

Munk, D., Kipouros, T., Vio, G., Steven, G., Parks, G., 2017a. Topology optimisation of micro fluidic mixers considering fluid-structure interactions with a coupled Lattice Boltzmann algorithm. Journal of Computational Physics 349, 11-32.

Munk, D., Vio, G., Kipouros, T., Parks, G., 2016. Computational design for micro fluidic devices using a tightly coupled lattice Boltzmann and level set-based optimization algorithm. In: Müller, J., Thompson, H., Duddeck, F., Toropov, V., Querin, O., Sienz, J. (Eds.), Proceedings of the 11th ASMO-UK/ISSMO/NOED2016 International Conference on Numerical Optimisation Methods for Engineering Design. 
Munk, D., Vio, G., Steven, G., 2015. Topology and shape optimization methods using evolutionary algorithms: A review. Structural and Multidisciplinary Optimization 52 (3), 613-631.

Munk, D., Vio, G., Steven, G., 2017b. A Bi-directional Evolutionary Structural Optimisation algorithm with an added connectivity constraint. Finite Elements in Analysis and Design 131, 25-42.

Osher, S., Santosa, F., 2001. Level set methods for optimization problems involving geometry and constraints. Journal of Computational Physics 171, $272-288$.

Osher, S., Sethian, J., 1988. Fronts propagating with curvature-dependent speed: Algorithms based on Hamiltion-Jacobi formulations. Journal of Computational Physics 79, 12-49.

Pan, C., Luo, L.-S., Miller, C. T., 2006. An evaluation of lattice Boltzmann schemes for porous medium flow simulations. Computers and Fluids 35, 898-909.

Pedersen, N., 2000. Maximization of eigenvalues using topology optimization. Structural and Multidisciplinary Optimization 20 (1), 2-11.

Picelli, R., van Dijk, R., Vicente, W., Pavanello, R., Langelaar, M., van Keulen, F., 2017. Topology optimization for submerged buoyant structures. Engineering Optimization 49 (1), 1-21.

Picelli, R., Vicente, W., Pavanello, R., 2015a. Bi-directional evolutionary structural optimization for design-dependent fluid pressure loading problems. Engineering Optimization 47 (10), 1324-1342.

Picelli, R., Vicente, W., Pavanello, R., Xie, Y., 2015b. Evolutionary topology optimization for natural frequency maximization problems considering acoustic-structure interaction. Finite Elements in Analysis and Design 106, 56-64.

Querin, O., Steven, G., Xie, Y., 1998. Evolutionary structural optimization (ESO) using a bi-directional algorithm. Engineering with Computers 15, 1034-1048. 
Rozvany, G., 2001. Aims, scope, methods, history and unified terminology of computer-aided topology optimization in structural mechanics. Structural and Multidisciplinary Optimization 21, 90-108.

Rozvany, G., 2009. A critical review of established methods of structural topology optimization. Structural and Multidisciplinary Optimization 37, 217-237.

Rozvany, G., Lewinski, T., 2014. Topology Optimization in Structural and Continuum Mechanics, 1st Edition. Berlin, Heidelberg, New York: Springer.

Rozvany, G., Zhou, M., Birker, T., 1992. Generalized shape optimization without homogenization. Structural Optimization 4 (3), 250-252.

Sethian, J., Wiegmann, A., 2000. Structural boundary design via level set and immersed interface methods. Journal of Computational Physics 163, 489-528.

Sigmund, O., 2001. Design of multiphysics actuators using topology optimization - part I: One-material structures. Computer Methods in Applied Mechanics and Engineering 190, 6577-6604.

Sigmund, O., Clausen, P., 2007. Topology optimization using a mixed formulation: An alternative way to solve pressure load problems. Computer Methods in Applied Mechanics and Engineering 196, 1874-1889.

Sigmund, O., Maute, K., 2013. Topology optimization approaches. Structural and Multidisciplinary Optimization 48, 1031-1055.

Sigmund, O., Petersson, J., 1998. Numerical instabilities in topology optimization: A survey on procedures dealing with checkerboards, meshdependencies and local minima. Structural Optimization 16, 68-75.

Steven, G., Li, Q., Xie, Y., 2000. Evolutionary topology and shape design for general physical field problems. Computational Mechanics 26 (2), 129-139.

Stolpe, M., Svanberg, K., 2001. An alternative interpolation scheme for mimimum compliance optimization. Structural and Multidisciplinary Optimization $22(2), 116-124$. 
Succi, S., 2001. The Lattice Boltzmann Equation for Fluid Dynamics and Beyond, 1st Edition. Oxford Science Publications.

Tsotskas, C., Kipouros, T., Savill, A., 2015. Fast multi-objective optimization of a micro-fluidic device by using graphics accelerators. Procedia Computer Science 51, 2237-2246.

van Dijk, N., Maute, K., Langelaar, M., van Keulen, F., 2013. Level-set methods for structural topology optimization: A review. Structural and Multidisciplinary Optimization 48, 437-472.

Wang, S., Wang, M., 2006. A moving superimposed finite element method for structural topology optimization. International Journal for Numerical Methods in Engineering 65, 1892-1922.

Xia, L., Xia, Q., Huang, X., Xie, Y., 2016. Bi-directional evolutionary structural optimization on advanced structures and materials: a comprehensive review. Archives of Computational Methods in Engineering, 1-42.

Xie, Y., Steven, G., 1993. A simple evolutionary procedure for structural optimization. Computers and Structures 49, 885-896.

Xie, Y., Steven, G., 1996. Evolutionary structural optimization for dynamical problems. Computers and Structures 58, 1067-1073.

Xie, Y., Steven, G., 1997. Evolutionary Structural Optimization, 1st Edition. Springer.

Yaji, K., Yamada, T., Yoshino, M., Matsumoto, T., Izui, K., Nishiwaki, S., 2014. Topology optimization using the lattice Boltzmann method incorporating level set boundary expression. Journal of Computational Physics $272,158-181$.

Yamada, T., Izui, K., Nishiwaki, S., Takezawa, A., 2010. A topology optimization method based on the level set method incorporating a fictitious interface energy. Computer Methods in Applied Mechanical Engineering 199, 2876-2891.

Yang, X., Xie, Y., Steven, G., 2005. Evolutionary methods for topology optimization of continuous structures with design dependent loads. Computers and Structures 83, 956-963. 
Zhang, H., Zhang, Z., Liu, S., 2008. A new boundary search scheme for topology optimization of continuum structures with design dependent loads. Structural and Multidisciplinary Optimization 37 (2), 121-129.

Zheng, B., Chang, C., Gea, H., 2009. Topology optimization with designdependent prssure loading. Structural and Multidisciplinary Optimization $38,535-543$.

Zhou, M., Rozvany, G., 1991. The COC algorithm, part II: Topological, geometry and generalized shape optimization. Computer Methods in Applied Mechanics and Engineering 89, 197-224.

Zhou, S., Li, Q., 2008. A variational level set method for the topology optimization of steady-state Navier-Stokes flow. Journal of Computational Physics 227, 10178-10195. 